\title{
Different faces of the shearlet group
}

\author{
Stephan Dahlke, \\ Sören Häuser $\stackrel{\ddagger}{\ddagger}$
}

\author{
Filippo De Mari \\ Gabriele Steidl;
}

September 11, 2018

\author{
Ernesto De Vito \\ Gerd Teschke $§$
}

\begin{abstract}
Recently, shearlet groups have received much attention in connection with shearlet transforms applied for orientation sensitive image analysis and restoration. The square integrable representations of the shearlet groups provide not only the basis for the shearlet transforms but also for a very natural definition of scales of smoothness spaces, called shearlet coorbit spaces. The aim of this paper is twofold: first we discover isomorphisms between shearlet groups and other well-known groups, namely extended Heisenberg groups and subgroups of the symplectic group. Interestingly, the connected shearlet group with positive dilations has an isomorphic copy in the symplectic group, while this is not true for the full shearlet group with all nonzero dilations. Indeed we prove the general result that there exist, up to adjoint action of the symplectic group, only one embedding of the extended Heisenberg algebra into the Lie algebra of the symplectic group.

Having understood the various group isomorphisms it is natural to ask for the relations between coorbit spaces of isomorphic groups with equivalent representations. These connections are examined in the second part of the paper. We describe how isomorphic groups with equivalent representations lead to isomorphic coorbit spaces. In particular we apply this result to square integrable representations of the connected shearlet groups and metaplectic representations of subgroups of the symplectic group. This implies the definition of metaplectic coorbit spaces.

Besides the usual full and connected shearlet groups we also deal with Toeplitz shearlet groups.
\end{abstract}

Keywords: Shearlet group, Heisenberg group, Symplectic group, Coorbit space theory

Mathematics Subject Classifiation 22D10, 22E30, 22E60, 42B35, 42C15

\footnotetext{
*FB12 Mathematik und Informatik, Philipps-Universität Marburg, Hans-Meerwein Straße, Lahnberge, 35032 Marburg, Germany, dahlke@mathematik.uni-kl.de

${ }^{\dagger}$ Dipartimento di Matematica, Università degli Studi di Genova, Via Dodecaneso 35, 16146 Genova, Italy, \{demari,devito\}@dima.unige.it

${ }^{\ddagger}$ Fachbereich für Mathematik, Technische Universität Kaiserslautern, Paul-Ehrlich-Str. 31, 67663 Kaiserslautern, Germany, \{haeuser,steidl\}@mathematik.uni-kl.de

$\S$ Institute for Computational Mathematics in Science and Technology, Hochschule Neubrandenburg, University of Applied Sciences, Brodaer Str. 2, 17033 Neubrandenburg, Germany, teschke@hs-nb.de
} 


\section{Introduction}

The shearlet transform was originally developed in the inaugural paper [31]. Among other transforms applied in the analysis of directional information, the continuous shearlet transform stands out because it is related to group theory, i.e., it can be derived from square integrable representations of the so-called shearlet group [9]. Recently, the shearlet transform and its modifications have found wide applications in the analysis and restoration of images, see, e.g., [21, 22, 23, 25, 28]. Further, the shearlet group and its square integrable representations give rise to a natural scale of smoothness spaces, so-called shearlet coorbit spaces [7, 10, 11] which can be considered as a special example of general coorbit spaces introduced by Feichtinger and Gröchenig [14, 15, 16]. An overview of recent developments on shearlets can be found in the book [30]. On the other hand, other groups such as the Heisenberg group or the symplectic group have been playing an important role in harmonic analysis, linear algebra and signal and image processing for a long time. In particular, the Heisenberg group is one of the basic tools for the mathematical foundation of the short-time Fourier transform, see [15, 19] for details. For the symplectic group we refer to [2, 3, 4] and references therein.

In this paper we ask for relations between the different groups and the corresponding coorbit spaces. In particular, we discover isometries of the connected shearlet group and its relatives to extended Heisenberg groups and subgroups of the symplectic group. Interestingly such results do not hold true for the full shearlet group. We show that isomorphic groups with equivalent representations give rise to equivalent coorbit spaces.

The first observation concerning the relationship between the extended Heisenberg group and the shearlet group is found in [32], where square integrability is shown, and it is recalled in the book [26]. The first embedding of the two-dimensional continuous shearlet group into $\operatorname{Sp}(2, \mathbb{R})$ is found in [27, and, with $\gamma=1$, implicitly appears in 2]. Similarly, the first embedding of shearlet-like groups with isotropic dilations in arbitrary dimensions (albeit with non-Toeplitz shearing matrices) into $\operatorname{Sp}(2, \mathbb{R})$ with matrices of the form $\Sigma \rtimes H$ is found in [27]. The isotropic shearlet-like groups were further explored in the paper [5], while the anisotropic case (including the embedding into $\operatorname{Sp}(2, \mathbb{R})$ ) was generalized to higher dimensions in [6].

Organization of the paper: In Section 2 we discover isomorphisms between shearlet and Toeplitz shearlet groups and other well-known groups. We show that the full and connected shearlet groups are isomorphic to full and connected extended Heisenberg groups, respectively. Further, we prove that the connected shearlet group is isomorphic to a subgroup of the symplectic group, which holds also true for the connected Toeplitz shearlet group. Section 3 deals with the full shearlet group. We show that it is not possible to embed this group into the symplectic group or in any of its coverings. The proof is based on the general result that the Lie algebra of the extended Heisenberg group can only be embedded in one way into the Lie algebra of the symplectic group. This result stands also for its own and is presented in Section 4 . Finally, in Section 5 the very natural relations between coorbit spaces of isomorphic groups with equivalent representations are presented. Naturally, these coorbit spaces are also isomorphic. At the end of Section 5 we use our findings to introduce metaplectic coorbit spaces. 


\section{Shearlet groups and their isomorphic relatives}

In this section we show that the shearlet groups are isomorphic to extended Heisenberg groups and that the connected shearlet group and Toeplitz shearlet group have an isomorphic subgroup within the symplectic group.

\subsection{Shearlet and Toeplitz shearlet groups}

For fixed $\gamma \in \mathbb{R}$ (usually $0<\gamma<1$, e.g., $\gamma=\frac{1}{d}$, to ensure directional selectivity) and $a \in \mathbb{R}^{*}:=\mathbb{R} \backslash\{0\}$ we introduce the dilation matrices

$$
A_{a, \gamma}:=\left(\begin{array}{cc}
a & 0 \\
0 & \operatorname{sgn}(a)|a|^{\gamma} I_{d-1}
\end{array}\right) \quad \text { and } \quad A_{a}:=a I_{d}
$$

and for $s=\left(s_{1}, \ldots, s_{d-1}\right)^{\mathrm{T}} \in \mathbb{R}^{d}$ the shear and Toeplitz shear matrices

$$
S_{s}:=\left(\begin{array}{cc}
1 & s^{\mathrm{T}} \\
0 & I_{d-1}
\end{array}\right) \quad \text { and } \quad T_{s}:=\left(\begin{array}{ccccc}
1 & s_{1} & s_{2} & \ldots & s_{d-1} \\
0 & 1 & s_{1} & s_{2} & \vdots \\
\vdots & \ddots & \ddots & \ddots & \vdots \\
\vdots & & \ddots & 1 & s_{1} \\
0 & \ldots & \ldots & 0 & 1
\end{array}\right) .
$$

Note that the product of two upper triangular Toeplitz matrices $T_{s}$ and $T_{s^{\prime}}$ is again an upper triangular Toeplitz matrix $T_{s \sharp s^{\prime}}$ with

$$
\left(s \sharp s^{\prime}\right)_{i}:=s_{i}+s_{i}^{\prime}+\sum_{j+k=i} s_{j}^{\prime} s_{k}, \quad i=1, \ldots, d-1 .
$$

The shearlet and Toeplitz shearlet groups are defined as follows:

- The (full) shearlet group $\mathbb{S}$ is the set $\mathbb{R}^{*} \times \mathbb{R}^{d-1} \times\left(\mathbb{R} \times \mathbb{R}^{d-1}\right)$ with the group operation

$$
(a, s, t) \circ_{\mathbb{S}}\left(a^{\prime}, s^{\prime}, t^{\prime}\right):=\left(a a^{\prime}, s+|a|^{1-\gamma} s^{\prime}, t+S_{s} A_{a, \gamma} t^{\prime}\right) .
$$

Using the notation $t=\left(t_{1}, \tilde{t}\right)^{\mathrm{T}} \in \mathbb{R}^{d}$ the group law can be rewritten as

$$
\left(a, s, t_{1}, \tilde{t}\right) \circ_{\mathbb{S}}\left(a^{\prime}, s^{\prime}, t_{1}^{\prime}, \tilde{t}^{\prime}\right)=\left(a a^{\prime}, s+|a|^{1-\gamma} s^{\prime}, t_{1}+a t_{1}^{\prime}+\operatorname{sgn}(a)|a|^{\gamma} s^{\mathrm{T}} \tilde{t}^{\prime}, \tilde{t}+\operatorname{sgn}(a)|a|^{\gamma} \tilde{t}^{\prime}\right) .
$$

- The connected shearlet group $\mathbb{S}^{+}$is the set $\mathbb{R}^{+} \times \mathbb{R}^{d-1} \times\left(\mathbb{R} \times \mathbb{R}^{d-1}\right)$ with group law (3) reduced to positive $a=|a|=\operatorname{sgn}(a)|a|$.

- The (full) Toeplitz shearlet group $\mathbb{S}_{T}$ is the set $\mathbb{R}^{*} \times \mathbb{R}^{d-1} \times \mathbb{R}^{d}$ with the group operation

$$
(a, s, t) \circ_{\mathbb{S}_{T}^{+}}\left(a^{\prime}, s^{\prime}, t^{\prime}\right)=\left(a a^{\prime}, s \sharp s^{\prime}, t+A_{a} T_{s} t^{\prime}\right) .
$$

- The connected Toeplitz shearlet group $\mathbb{S}_{T}^{+}$is the set $\mathbb{R}^{+} \times \mathbb{R}^{d-1} \times \mathbb{R}^{d}$ with group law (4) restricted to positive $a$. 
The four groups are locally compact groups, where the left and right Haar measures of the shearlet groups are given by

$$
d \mu_{\mathbb{S}, l}(a, s, t):=\frac{1}{|a|^{d+1}} d a d s d t \quad \text { and } \quad d \mu_{\mathbb{S}, r}(a, s, t):=\frac{1}{|a|} d a d s d t .
$$

see [9, 11] and of the Toeplitz shearlet groups by

$$
d \mu_{\mathbb{S}_{T}, l}(a, s, t)=\frac{1}{|a|^{d+1}} d a d s d t \quad \text { and } \quad d \mu_{\mathbb{S}_{T}, r}(a, s, t)=\frac{1}{|a|} d a d s d t
$$

see [8, 12] with restriction to positive dilations $a$ for the connected groups. The connected (Toeplitz) shearlet group is a subgroup of the the full (Toeplitz) shearlet group.

\subsection{Relation to Heisenberg groups}

The Heisenberg group and its polarized version are defined as follows:

- The Heisenberg group $\mathbb{H}$ is the set $\mathbb{R}^{d-1} \times \mathbb{R} \times \mathbb{R}^{d-1}$ endowed with the group operation

$$
(p, \tau, q) \circ_{\mathbb{H}}\left(p^{\prime}, \tau^{\prime}, q^{\prime}\right):=\left(p+p^{\prime}, \tau+\tau^{\prime}+\frac{1}{2}\left(p^{\mathrm{T}} q^{\prime}-q^{\mathrm{T}} p^{\prime}\right), q+q^{\prime}\right) .
$$

- The polarized Heisenberg group $\mathbb{H}^{\mathrm{pol}}$ is the same set $\mathbb{R}^{d-1} \times \mathbb{R} \times \mathbb{R}^{d-1}$ but with the group operation

$$
(p, \tau, q) \diamond_{\mathbb{H} \text { pol }}\left(p^{\prime}, \tau^{\prime}, q^{\prime}\right):=\left(p+p^{\prime}, \tau+\tau^{\prime}+p^{\mathrm{T}} q^{\prime}, q+q^{\prime}\right) .
$$

These Heisenberg groups are isomorphic with isomorphism given by

$$
\phi: \mathbb{H} \rightarrow \mathbb{H}^{\mathrm{pol}}, \quad(p, \tau, q) \mapsto\left(p, \tau+\frac{1}{2} p^{\mathrm{T}} q, q\right) .
$$

This is why we usually write the Heisenberg group. If we set $a=a^{\prime}=1$ in (3) we obtain

$$
\left(1, s, t_{1}, \tilde{t}\right) \circ_{\mathbb{S}}\left(1, s^{\prime}, t_{1}^{\prime}, \tilde{t}^{\prime}\right)=\left(1, s+s^{\prime}, t_{1}+t_{1}^{\prime}+s^{\mathrm{T}} \tilde{t}^{\prime}, \tilde{t}+\tilde{t}^{\prime}\right)
$$

which looks very similar to the group law of the Heisenberg group in (5). We will show that the shearlet group is isomorphic to an extended Heisenberg group which is equipped with a dilation. For the general concept of group extensions we refer to [29]. We briefly recall the notion of a semi-direct product. Given a group $H$ and a group $G$ acting on $H$ by automorphisms, i.e., a smooth map $\delta: G \times H \rightarrow H$ is defined such that $\delta(g, \cdot)$ is an automorphism of $H$, we can extend $H$ by $G$ by forming the semi-direct product $H \rtimes G$. The multiplication and inversion are determined by

$$
(h, g)\left(h^{\prime}, g^{\prime}\right)=\left(h \circ_{H} \delta\left(g, h^{\prime}\right), g \circ_{G} g^{\prime}\right) \quad \text { and } \quad(h, g)^{-1}=\left(\delta\left(g^{-1}, h^{-1}\right), g^{-1}\right) .
$$

The extended Heisenberg group is the semi-direct product of the Heisenberg group $\mathbb{H}$ and $\mathbb{R}^{*}$, where $\mathbb{R}^{*}$ acts on $\mathbb{H}$ via the automorphism

$$
\delta_{a}^{\gamma}(p, \tau, q):=\left(|a|^{1-\gamma} p, a \tau, \operatorname{sgn}(a)|a|^{\gamma} q\right), \quad \gamma>0,
$$

for details see [24]. In other words, 
- the extended Heisenberg group is defined by $\mathbb{H}_{e}:=\mathbb{H} \rtimes \mathbb{R}^{*}$ with the group operation

$$
\begin{aligned}
&(p, \tau, q, a) \mathrm{OH}_{\mathbb{H}_{e}}\left(p^{\prime}, \tau^{\prime}, q^{\prime}, a^{\prime}\right):= \\
&\left(p+|a|^{1-\gamma} p^{\prime}, \tau+a \tau^{\prime}+\frac{1}{2}\left(\operatorname{sgn}(a)|a|^{\gamma} p^{\mathrm{T}} q^{\prime}-|a|^{1-\gamma} q^{\mathrm{T}} p^{\prime}\right), q+\operatorname{sgn}(a)|a|^{\gamma} q^{\prime}, a a^{\prime}\right)
\end{aligned}
$$

- and the extended polarized Heisenberg group by $\mathbb{H}_{e}^{\mathrm{pol}}:=\mathbb{H}^{\mathrm{pol}} \rtimes \mathbb{R}^{*}$ with group operation

$$
(p, \tau, q, a) \circ_{\mathbb{H}_{e}^{\mathrm{pol}}}\left(p^{\prime}, \tau^{\prime}, q^{\prime}, a^{\prime}\right):=\left(p+|a|^{1-\gamma} p^{\prime}, \tau+a \tau^{\prime}+\operatorname{sgn}(a)|a|^{\gamma} p^{\mathrm{T}} q^{\prime}, q+\operatorname{sgn}(a)|a|^{\gamma} q^{\prime}, a a^{\prime}\right) .
$$

These groups are again isomorphic with

$$
\phi_{e}: \mathbb{H}_{e} \rightarrow \mathbb{H}_{e}^{\mathrm{pol}}, \quad(p, \tau, q, a) \mapsto\left(p, \tau+\frac{1}{2} p^{\mathrm{T}} q, q, a\right) .
$$

Using only positive dilations we obtain the connected versions of the extended (polarized) Heisenberg group, whose composition laws we do not write explicitly. For $\gamma=\frac{1}{2}$ the dilation is symmetric in $p$ and $q$. Comparing the definition of $\mathbb{S}$ and $\mathbb{H}_{e}^{\text {pol }}$ we see that both groups coincide up to a permutation of the variables. The same holds true for the connected group variants. Taking further the isomorphism between the Heisenberg groups and its polarized versions into account we can summarize:

Lemma 2.1. (Relation between extended Heisenberg groups and shearlet groups) The following relations hold true:

$$
\mathbb{H}_{e} \cong \mathbb{H}_{e}^{\mathrm{pol}}=\mathbb{S} \quad \text { and } \quad \mathbb{H}_{e}^{+} \cong \mathbb{H}_{e}^{\mathrm{pol},+}=\mathbb{S}^{+} .
$$

\subsection{Relation to subgroups of the symplectic group}

Let $\operatorname{GL}(d, \mathbb{R})$ denote the general linear group of real, invertible $d \times d$ matrices. The symplectic group $\operatorname{Sp}(d, \mathbb{R})$ is the group of all matrices $B \in \mathrm{GL}(2 d, \mathbb{R})$ fulfilling $B^{\mathrm{T}} J B=J$ for $J=\left(\begin{array}{cc}0 & I_{d} \\ -I_{d} & 0\end{array}\right)$, i.e.,

$$
\operatorname{Sp}(d, \mathbb{R}):=\left\{B \in \mathbb{R}^{2 d \times 2 d}: B^{\mathrm{T}} J B=J\right\} .
$$

Let $H$ be a closed subgroup of $\operatorname{GL}(d, \mathbb{R})$ and $\Sigma$ an additive subspace of the symmetric matrices $\operatorname{Sym}(d, \mathbb{R})$ that is invariant under the $H$-action given by $M^{-\mathrm{T}} \sigma M^{-1} \in \Sigma$ for all $M \in H$ and $\sigma \in \Sigma$. Then we know by [13, Example 3] that the semi-direct product

$$
\Sigma \rtimes H:=\left\{\left(\begin{array}{cc}
M & 0 \\
\sigma M & M^{-\mathrm{T}}
\end{array}\right): M \in H, \sigma \in \Sigma\right\}
$$

is a subgroup of $\operatorname{Sp}(d, \mathbb{R})$. We are interested in two special groups of the form (7). The first one is the group $\operatorname{TDS}(d)$ of translations, dilations and shears defined by

$$
\operatorname{TDS}(d):=\left\{\left(\begin{array}{cc}
M(s, a) & 0 \\
\sigma(t) M(s, a) & M(s, a)^{-\mathrm{T}}
\end{array}\right): a \in \mathbb{R}^{+}, s \in \mathbb{R}^{d-1}, t \in \mathbb{R}^{d}\right\},
$$

where

$$
\widetilde{A}_{a, \gamma}:=\left(\begin{array}{cc}
a^{-\frac{1}{2}} & 0 \\
0 & a^{\frac{1}{2}-\gamma} I_{d-1}
\end{array}\right), \quad \widetilde{S}_{s}:=\left(\begin{array}{cc}
1 & 0 \\
-s & I_{d-1}
\end{array}\right), \quad M(s, a):=\widetilde{S}_{s} \widetilde{A}_{a, \gamma}
$$


and $\sigma(t)$ belongs to the subspace of the symmetric matrices

$$
\left\{\sigma(t)=\sigma\left(t_{1}, \tilde{t}\right):=\left(\begin{array}{cc}
t_{1} & \frac{1}{2} \tilde{t}^{T} \\
\frac{1}{2} \tilde{t} & 0_{d-1, d-1}
\end{array}\right): t \in \mathbb{R}^{d}\right\} .
$$

Straightforward computation shows that the subspace (9) is indeed invariant under $H$-action with matrices $M(s, a)$.

The relation between this subgroup $\operatorname{TDS}(d)$ of the symplectic group and the connected shearlet group $\mathbb{S}^{+}$is stated in the following lemma. For a proof we refer to [24, 13].

Lemma 2.2. (Relation between $\mathbb{S}^{+}$and $\operatorname{TDS}(d)$ )

The groups $\mathbb{S}^{+}$and $\operatorname{TDS}(d)$ are isomorphic and the isomorphism $\kappa^{+}$is given by

$$
\kappa^{+}: \mathbb{S}^{+} \rightarrow \operatorname{TDS}(d), \quad\left(a, s, t_{1}, \tilde{t}\right) \mapsto\left(\begin{array}{cc}
M(s, a) & 0 \\
\sigma\left(t_{1}, \tilde{t}\right) M(s, a) & M(s, a)^{-T}
\end{array}\right) .
$$

The second interesting group of the form (77) is the group of translations, dilations and Toeplitz shears given by

$$
\operatorname{TDS}_{T}(d)=\left\{\left(\begin{array}{cc}
a^{-1 / 2} T_{s}^{-\mathrm{T}} & 0 \\
a^{-1 / 2} \sigma(t) T_{s}^{-\mathrm{T}} & a^{1 / 2} T_{s}
\end{array}\right): a \in \mathbb{R}^{+}, s \in \mathbb{R}^{d-1}, t \in \mathbb{R}^{d}\right\} .
$$

Clearly, the subspace (9) is invariant under the $H$-action with matrices $a^{-1 / 2} T_{s}^{-1}$. This group is related to the connected Toeplitz shearlet group as follows:

Lemma 2.3. (Relation between $\mathbb{S}_{T}^{+}$and $\operatorname{TDS}_{T}(d)$ )

The groups $\mathbb{S}_{T}^{+}$and $\operatorname{TDS}_{T}(d)$ are isomorphic and the isomorphism $\kappa_{T}^{+}$is given by

$$
\kappa_{T}^{+}: \mathbb{S}_{T}^{+} \rightarrow \operatorname{TDS}_{T}(d), \quad\left(a, s, t_{1}, \tilde{t}\right) \mapsto\left(\begin{array}{cc}
a^{-1 / 2} T_{s}^{-T} & 0 \\
a^{-1 / 2} \sigma\left(t_{1}, \tilde{t}\right) T_{s}^{-T} & a^{1 / 2} T_{s}
\end{array}\right) .
$$

Proof. From the group law in the Toeplitz shearlet group we know that

$$
(a, s, t) \circ_{\mathbb{S}_{T}^{+}}\left(a^{\prime}, s^{\prime}, t^{\prime}\right)=\left(a a^{\prime}, s \sharp s^{\prime}, t+a T_{s} t^{\prime}\right),
$$

so that we have to show

$$
\kappa_{T}^{+}(a, s, t) \circ \kappa_{T}^{+}\left(a^{\prime}, s^{\prime}, t^{\prime}\right)=\kappa_{T}^{+}\left(a a^{\prime}, s \sharp s^{\prime}, t+a T_{s} t^{\prime}\right) .
$$

The right hand side can be rewritten as

$$
\kappa_{T}^{+}\left(a a^{\prime}, s \sharp s^{\prime}, t+a T_{s} t^{\prime}\right)=\left(\begin{array}{cc}
\left(a a^{\prime}\right)^{-\frac{1}{2}} T_{s \sharp s^{\prime}}^{-\mathrm{T}} & 0 \\
\left(a a^{\prime}\right)^{-\frac{1}{2}} \sigma\left(t_{1}+a\left(t_{1}^{\prime}+s^{\mathrm{T}} \tilde{t}^{\prime}\right), \tilde{t}+a T_{[s]} \tilde{t}^{\prime}\right) T_{s \sharp s^{\prime}}^{-\mathrm{T}} & \left(a a^{\prime}\right)^{\frac{1}{2}} T_{s \sharp s^{\prime}}
\end{array}\right)
$$

where $[s]=\left(s_{i}\right)_{i=1}^{d-2} \in \mathbb{R}^{d-2}$. For the left hand side we have

$$
\begin{aligned}
& \left(\begin{array}{cc}
a^{-1 / 2} T_{s}^{-\mathrm{T}} & 0 \\
a^{-1 / 2} \sigma\left(t_{1}, \tilde{t}\right) T_{s}^{-\mathrm{T}} & a^{1 / 2} T_{s}
\end{array}\right)\left(\begin{array}{cc}
\left(a^{\prime}\right)^{-1 / 2} T_{s^{\prime}}^{-\mathrm{T}} & 0 \\
\left(a^{\prime}\right)^{-1 / 2} \sigma\left(t_{1}^{\prime}, \tilde{t}^{\prime}\right) T_{s^{\prime}}^{-\mathrm{T}} & \left(a^{\prime}\right)^{1 / 2} T_{s^{\prime}}
\end{array}\right) \\
& =\left(\begin{array}{cc}
\left(a a^{\prime}\right)^{-1 / 2} T_{s \sharp s^{\prime}}^{-\mathrm{T}} & 0 \\
\left(a a^{\prime}\right)^{-1 / 2}\left(\sigma\left(t_{1}, \tilde{t}\right)+a T_{s} \sigma\left(t_{1}^{\prime}, \tilde{t}^{\prime}\right) T_{s}^{\mathrm{T}}\right) T_{s \sharp s^{\prime}}^{-\mathrm{T}} & \left(a a^{\prime}\right)^{1 / 2} T_{s \sharp s^{\prime}}
\end{array}\right)
\end{aligned}
$$


consequently, it is sufficient to show

$$
\left(\begin{array}{cc}
t_{1}^{\prime}+s^{\mathrm{T}} \tilde{t}^{\prime} & \frac{1}{2}\left(T_{[s]} \tilde{t}^{\prime}\right)^{\mathrm{T}} \\
\frac{1}{2} T_{[s]} \tilde{t}^{\prime} & 0
\end{array}\right)=T_{s}\left(\begin{array}{cc}
t_{1}^{\prime} & \frac{1}{2}\left(\tilde{t}^{\prime}\right)^{\mathrm{T}} \\
\frac{1}{2} \tilde{t}^{\prime} & 0
\end{array}\right) T_{s}^{\mathrm{T}} .
$$

The right hand side of (12) is

$$
\begin{aligned}
& \left(\begin{array}{cccc}
1 & s_{1} & \cdots & s_{d-1} \\
& \ddots & & \vdots \\
& & \ddots & s_{1} \\
& & & 1
\end{array}\right)\left(\begin{array}{cc}
t_{1}^{\prime} & \frac{1}{2}\left(\tilde{t}^{\prime}\right)^{\mathrm{T}} \\
\frac{1}{2} \tilde{t}^{\prime} & 0
\end{array}\right)\left(\begin{array}{cccc}
1 & & & \\
s_{1} & \ddots & & \\
\vdots & & \ddots & \\
s_{d-1} & \cdots & s_{1} & 1
\end{array}\right) \\
& =\left(\begin{array}{cc}
t_{1}^{\prime}+\frac{1}{2} s^{\mathrm{T}} \tilde{t}^{\prime} & \frac{1}{2}\left(\tilde{t}^{\prime}\right)^{\mathrm{T}} \\
\frac{1}{2} T_{[s]} \tilde{t}^{\prime} & 0
\end{array}\right)\left(\begin{array}{cccc}
1 & & & \\
s_{1} & \ddots & & \\
\vdots & & \ddots & \\
s_{d-1} & \cdots & s_{1} & 1
\end{array}\right)=\left(\begin{array}{cc}
t_{1}^{\prime}+\frac{1}{2} s^{\mathrm{T}} \tilde{t}^{\prime}+\frac{1}{2} s^{\mathrm{T}} \tilde{t}^{\prime} & \frac{1}{2}\left(T_{[s]} \tilde{t}^{\prime}\right)^{\mathrm{T}} \\
\frac{1}{2} T_{[s]} \tilde{t}^{\prime} & 0
\end{array}\right)
\end{aligned}
$$

which coincides with the left hand side of (12) and we are done.

\section{Embedding of the full shearlet group}

In the following we want to prove that it is not possible to embed the full shearlet group into the symplectic group $\operatorname{Sp}(2, \mathbb{R})$ or one of its covers. To show this result we pursuit the following path: a) establish a necessary property for those continuous, injective group homomorphisms of $\mathbb{S}^{+}$to $\operatorname{Sp}(d, \mathbb{R})$ which can be extended to $\mathbb{S}$; b) show that this property is not fulfilled by the special homomorphism $\kappa^{+}$defined in (10) nor by its conjugation or concatenations with isomorphisms of $\mathbb{S}^{+}$; c) prove that in dimension $d=2$ actually any continuous, injective group homomorphism is given up to conjugation or concatenations with isomorphisms of $\mathbb{S}^{+}$by the $\operatorname{map} \kappa^{+}$.

In the following $\mathbb{S}$ is regarded as the semi-direct product of its closed normal subgroup $\mathbb{S}^{+}$and its finite subgroup $\{( \pm 1,0,0,0)\} \simeq \mathbb{Z}_{2}$. Indeed,

$$
(-1,0,0,0) \circ_{\mathbb{S}}\left(a, s, t_{1}, \tilde{t}\right) \circ_{\mathbb{S}}(-1,0,0,0)=\left(a, s,-t_{1},-\tilde{t}\right)
$$

and, clearly,

$$
\mathbb{S}^{+} o_{\mathbb{S}} \mathbb{Z}_{2}=\mathbb{S} \text { and } \mathbb{S}^{+} \cap \mathbb{Z}_{2}=\{(1,0,0,0)\} .
$$

With slight abuse of notation, we write an element of $\mathbb{S}$ as a pair $(x, \varepsilon)$ where $x=\left(a, s, t_{1}, \tilde{t}\right) \in \mathbb{S}^{+}$ and $\varepsilon \in \mathbb{Z}_{2}$. The group operation in $\mathbb{S}$ becomes

$$
(x, \varepsilon) \circ_{\mathbb{S}}\left(x^{\prime}, \varepsilon^{\prime}\right)=\left(x \circ_{\mathbb{S}} R_{\varepsilon} x^{\prime}, \varepsilon \varepsilon^{\prime}\right),
$$

where $R_{\varepsilon}$ is the group isomorphism of $\mathbb{S}^{+}$given by

$$
R_{\varepsilon} x=R_{\varepsilon}\left(a, s, t_{1}, \tilde{t}\right)=\left(a, s, \varepsilon t_{1}, \varepsilon \tilde{t}\right) .
$$

Let $e:=(1,0,0,0)$ denote the identity of $\mathbb{S}^{+}$. Then, in particular,

$$
(e,-1) \circ_{\mathbb{S}}(x, 1)=\left(R_{-1} x, 1\right) \circ_{\mathbb{S}}(e,-1)
$$

and $(e, 1)$ is the identity of $\mathbb{S}$. 
Lemma 3.1. An injective group homomorphism $g^{+}: \mathbb{S}^{+} \rightarrow \operatorname{Sp}(d, \mathbb{R})$ extends to a group homomorphism $g: \mathbb{S} \rightarrow \operatorname{Sp}(d, \mathbb{R})$ if and only if there exists $A \in \operatorname{Sp}(d, \mathbb{R})$ such that

$$
A^{2}=I_{2 d} \quad \text { and } \quad \mathrm{Ag}^{+}(x)=g^{+}\left(R_{-1} x\right) A
$$

for all $x \in \mathbb{S}^{+}$. Under these assumptions we have for all $x \in \mathbb{S}^{+}$that

$$
g(x, 1)=g^{+}(x) \text { and } g(x,-1)=g^{+}(x) A .
$$

The extended group homomorphism $g$ is injective.

Note that by injectivity of $g^{+}$we have $A \neq I$ for any $A$ fulfilling (15).

Proof. $\Rightarrow$ : Assume that $g^{+}: \mathbb{S}^{+} \rightarrow \operatorname{Sp}(d, \mathbb{R})$ extends to a homomorphism $g: \mathbb{S} \rightarrow \operatorname{Sp}(d, \mathbb{R})$. Then we have in particular $g(x, 1)=g^{+}(x)$ for all $x \in \mathbb{S}^{+}$. We show that $A:=g(e,-1)$ fulfills (15). Since $(e,-1) \circ_{\mathbb{S}}(e,-1)=\left(e \circ_{\mathbb{S}^{+}} R_{-1} e, 1\right)=(e, 1)$ and $g$ is a homomorphism we obtain $A^{2}=I_{2 d}$. By

$$
(e,-1) \circ_{\mathbb{S}}(x, 1)=\left(e \circ_{\mathbb{S}^{+}} R_{-1} x,-1\right)=\left(R_{-1} x, 1\right) \circ_{\mathbb{S}}(e,-1)
$$

and the fact that $g$ is a homomorphism we get the second equality in (15). Finally, we conclude since $(x,-1) \circ_{\mathbb{S}}(e,-1)=\left(x \circ_{\mathbb{S}} R_{-1} e, 1\right)=(x, 1), g$ is a homomorphism and $A^{2}=I_{2 d}$ that $g(x,-1)=g^{+}(x) A$.

$\Leftarrow$ : Conversely, assume that there exists $A \in \operatorname{Sp}(d, \mathbb{R})$ satisfying (15). Set

$$
g(x, \varepsilon):= \begin{cases}g^{+}(x) & \text { for } \varepsilon=1, \\ g^{+}(x) A & \text { for } \varepsilon=-1 .\end{cases}
$$

Then (16) is fulfilled by definition. Direct computation shows that $g$ is a group homomorphism from $\mathbb{S}$ into $\operatorname{Sp}(d, \mathbb{R})$.

It remains to prove the injectivity of $g$. By (16) and the invertibility of $A$ we see that $g(x, \varepsilon)=$ $g\left(x^{\prime}, \varepsilon\right)$ implies $g^{+}(x)=g^{+}\left(x^{\prime}\right)$ and since $g^{+}$is injective further $x=x^{\prime}$. If $g\left(x^{\prime}, 1\right)=g(x,-1)$ we obtain $g^{+}\left(x^{\prime}\right)=g^{+}(x) A$ and since $g^{+}$is a homomorphism that $g^{+}\left(x^{-1} x^{\prime}\right)=A$. Set $y:=x^{-1} x^{\prime} \in \mathbb{S}^{+}$. Since $g^{+}$is a homomorphism we conclude $g^{+}\left(y^{2}\right)=\left(g^{+}(y)\right)^{2}=A^{2}=I_{2 d}$ and with the injectivity of $g^{+}$that $y^{2}=e$. But this is only possible if $y=e$ and consequently $g^{+}(y)=I_{2 d}=A$ which is a contradiction. Hence $g$ is injective.

We recall that a covering group of $\operatorname{Sp}(d, \mathbb{R})$ is a connected Lie group $G$ with a surjective continuous group homomorphism $p: G \rightarrow \operatorname{Sp}(d, \mathbb{R})$ whose kernel is discrete.

Lemma 3.2. Let $(G, p)$ be a covering group of $\operatorname{Sp}(d, \mathbb{R})$ with covering homomorphism $p$ and an injective continuous group homomorphism $i: \mathbb{S} \rightarrow G$. Then $p \circ i$ is an injective group homomorphism of $\mathbb{S}$ into $\operatorname{Sp}(d, \mathbb{R})$.

Proof. By definition it is clear that $p \circ i$ is a homomorphism. Next we have that $p \circ i$ restricted to $\mathbb{S}^{+}$is injective by the following argument. Recall that a continuous group homomorphism of a Lie group is always smooth. Since $\mathbb{S}^{+}$is a connected, simply connected Lie group it is enough to prove that its tangent map at the identity $\left.(p \circ i)_{*}\right|_{e}$ is injective. Observe that $\left.(p \circ i)_{*}\right|_{e}=\left.\left.p_{*}\right|_{e_{G}} i_{*}\right|_{e}$. Since $i$ is injective, the same holds true for $\left.i_{*}\right|_{e}$ and since $p$ is a covering homomorphism $\left.p_{*}\right|_{e_{G}}$ is injective. Thus their concatenation is injective. Now applying Lemma 3.1 with $g^{+}:=\left.p \circ i\right|_{\mathbb{S}^{+}}$yields the assertion. 
We have shown that a special injective homomorphism from $\mathbb{S}^{+}$into $\operatorname{Sp}(2, \mathbb{R})$ is given by $g^{+}:=\kappa^{+}$defined in (10).

Lemma 3.3. For $\kappa^{+}: \mathbb{S}^{+} \rightarrow \operatorname{Sp}(d, \mathbb{R})$ defined by (10) there does not exist $A \in \operatorname{Sp}(d, \mathbb{R})$ satisfying (15). The same holds true for

(i) any conjugation of $\kappa^{+}$, i.e., for any map $\kappa_{B}^{+}: \mathbb{S}^{+} \rightarrow \operatorname{Sp}(d, \mathbb{R})$ with $\kappa_{B}^{+}(x):=B \kappa^{+}(x) B^{-1}$, $B \in \operatorname{Sp}(d, \mathbb{R})$,

(ii) any map $\kappa_{\varphi}^{+}: \mathbb{S}^{+} \rightarrow \operatorname{Sp}(d, \mathbb{R})$ of the form $\kappa_{\varphi}^{+}(x):=\kappa^{+}(\varphi(x))$, where $\varphi$ is a group automorphism of $\mathbb{S}^{+}$such that

$$
\varphi\left(R_{-1} x\right)=R_{-1} \varphi(x)
$$

for all $x \in \mathbb{S}^{+}$.

Proof. 1. Assume that there exists $A:=\left(\begin{array}{cc}\alpha & \beta \\ \gamma & \delta\end{array}\right) \in \operatorname{Sp}(d, \mathbb{R})$ satisfying (15) for $\kappa^{+}$.

Since $A$ is symplectic it holds $A^{\mathrm{T}} J A=J$ and since $A^{-1}=A$ further $A^{\mathrm{T}} J=J A$. Hence

$$
\left(\begin{array}{ll}
\alpha^{\mathrm{T}} & \gamma^{\mathrm{T}} \\
\beta^{\mathrm{T}} & \delta^{\mathrm{T}}
\end{array}\right)\left(\begin{array}{cc}
0 & I_{d} \\
-I_{d} & 0
\end{array}\right)=\left(\begin{array}{cc}
-\gamma^{\mathrm{T}} & \alpha^{\mathrm{T}} \\
-\delta^{\mathrm{T}} & \beta^{\mathrm{T}}
\end{array}\right)=\left(\begin{array}{cc}
0 & I_{d} \\
-I_{d} & 0
\end{array}\right)\left(\begin{array}{cc}
\alpha & \beta \\
\gamma & \delta
\end{array}\right)=\left(\begin{array}{cc}
\gamma & \delta \\
-\alpha & -\beta
\end{array}\right)
$$

which implies $\beta=-\beta^{\mathrm{T}}, \gamma=-\gamma^{\mathrm{T}}$ and $\delta=\alpha^{\mathrm{T}}$. Thus, $A=\left(\begin{array}{ll}\alpha & \beta \\ \gamma & \alpha^{\mathrm{T}}\end{array}\right)$ with skew-symmetric $\beta, \gamma$. In particular, $\beta$ and $\gamma$ have zero diagonal elements. The second condition in (15) with $x:=\left(1,0, t_{1}, \tilde{t}\right)$ results by definition of $\kappa^{+}$in

$$
A\left(\begin{array}{cc}
I_{d} & 0 \\
\sigma\left(t_{1}, \tilde{t}\right) & I_{d}
\end{array}\right)=\left(\begin{array}{cc}
I_{d} & 0 \\
-\sigma\left(t_{1}, \tilde{t}\right) & I_{d}
\end{array}\right) A
$$

and straightforward computation shows that this implies

$$
\begin{aligned}
\beta \sigma\left(t_{1}, \tilde{t}\right) & =0=\sigma\left(t_{1}, \tilde{t}\right) \beta, \\
\alpha^{\mathrm{T}} \sigma\left(t_{1}, \tilde{t}\right) & =-\sigma\left(t_{1}, \tilde{t}\right) \alpha
\end{aligned}
$$

for all $t \in \mathbb{R}^{d}$. Since $\beta=-\beta^{\mathrm{T}}$, it has the form $\beta=\left(\begin{array}{cc}0 & -u^{\mathrm{T}} \\ u & M\end{array}\right)$ with $u \in \mathbb{R}^{d-1}$ and $M^{\mathrm{T}}=-M \in$ $\mathbb{R}^{d-1 \times d-1}$. Choosing $t:=(1,0, \ldots, 0)$ in (18) we see immediately by definition (9) of $\sigma$ that $u=0$. Let $\alpha=\left(\begin{array}{ll}a & w^{\mathrm{T}} \\ v & N\end{array}\right)$ with $v, w \in \mathbb{R}^{d}$ and $N \in \mathbb{R}^{d-1 \times d-1}$. Evaluating (19) for $t:=(1,0, \ldots, 0)$ we obtain $a=0$ and $w=0$. In summary, the matrix $A$ is of the form

$$
A=\left(\begin{array}{cccc}
0 & \mathbf{0}^{\mathrm{T}} & 0 & \mathbf{0}^{\mathrm{T}} \\
v & N & \mathbf{0} & M \\
& & 0 & v^{\mathrm{T}} \\
& \gamma & \mathbf{0} & N^{\mathrm{T}}
\end{array}\right) .
$$

Evidently, $A$ is not invertible because it has a zero column.

2. (i) Assume that there exists $A \in \operatorname{Sp}(d, \mathbb{R})$ satisfying (15) for some conjugation map $\kappa_{B}^{+}$. But then $\tilde{A}:=B^{-1} A B \in \operatorname{Sp}(d, \mathbb{R})$ fulfills (15) for $\kappa^{+}$. This contradicts the first part of the proof.

(ii) Finally, assume there exists $A \in \operatorname{Sp}(d, \mathbb{R})$ such that $A \kappa_{\varphi}^{+}(y) A^{-1}=\kappa_{\varphi}^{+}\left(R_{-1} y\right)$ for all $y \in \mathbb{S}^{+}$. With $y=\varphi^{-1}(x)$ we get $A \kappa^{+}(y) A^{-1}=\kappa^{+}\left(R_{-1} y\right)$, which is again a contradiction. 
Lemma 3.1 and Lemma 3.3 imply that the special group homomorphism $\kappa^{+}: \mathbb{S}^{+} \rightarrow \operatorname{Sp}(d, \mathbb{R})$ in (10) as well as its conjugations or concatenations with group automorphisms of $\mathbb{S}^{+}$satisfying (17) cannot be extended to a group homomorphism of $\mathbb{S}$ to $\operatorname{Sp}(d, \mathbb{R})$. For $d=2$ we have the sharper result that this holds true for all injective continuous group homomorphisms $g^{+}: \mathbb{S}^{+} \rightarrow$ $\operatorname{Sp}(2, \mathbb{R})$. The following theorem will be proved in the next section.

Theorem 3.4. Let $\gamma \in(0,1) \backslash\left\{\frac{1}{3}, \frac{2}{3}\right\}$. For any injective continuous group homomorphism $g^{+}: \mathbb{S}^{+} \rightarrow \mathrm{Sp}(2, \mathbb{R})$ there exists $B \in \mathrm{Sp}(2, \mathbb{R})$ and a continuous group isomorphism $\varphi$ of $\mathbb{S}^{+}$ satisfying (17) such that

$$
g^{+}(x)=B \kappa^{+}(\varphi(x)) B^{-1} .
$$

As immediate consequence of the theorem, Lemma 3.1 and Lemma 3.3 we obtain our main result (for $\gamma=\frac{1}{3}$ and $\gamma=\frac{2}{3}$ see Remark 4.3).

Theorem 3.5. Let $\gamma \in(0,1)$. There does not exist an injective continuous homomorphism from $\mathbb{S}$ into $\operatorname{Sp}(2, \mathbb{R})$ and into any of its coverings.

We state the above result for $\gamma \in(0,1)$ since this is the range of interest in the applications. However, a simple inspection of the proof of Theorem 3.5 shows that Theorem 3.4 holds true for any $\gamma \in \mathbb{R} \backslash\{0,1\}$.

It is not clear if the theorem can be generalized to higher dimensions $d>2$. However, we conjecture that the result holds true in arbitrary dimensions.

\section{Proof of Theorem 3.4}

We start by examining the Lie algebra of the symplectic group in the next subsection and use the findings for our embedding result in Subsection 4.2 .

\subsection{Root space decomposition and canonical forms}

The Lie algebra $\mathfrak{s p}(2, \mathbb{R})$ of the symplectic group $\operatorname{Sp}(2, \mathbb{R})$ consists of the real $4 \times 4$ matrices, called Hamiltonians, which satisfy the equation $X^{\mathrm{T}} J+J X=0$. It is the 10-dimensional Lie algebra

$$
\mathfrak{s p}(2, \mathbb{R})=\left\{\left(\begin{array}{cc}
M_{11} & M_{12} \\
M_{21} & -M_{11}^{\mathrm{T}}
\end{array}\right): M_{11} \in \mathbb{R}^{2 \times 2}, M_{12}, M_{21} \in \operatorname{Sym}(2, \mathbb{R})\right\} .
$$

Root space decomposition. To prove our main embedding result we need a representation of Hamiltonians with respect to a certain basis of $\mathfrak{s p}(2, \mathbb{R})$ which we provide next. The maximally non compact Cartan subalgebra of $\mathfrak{s p}(2, \mathbb{R})$ is given by

$$
\mathfrak{a}:=\left\{H_{a, b}:=\left(\begin{array}{cccc}
a & 0 & 0 & 0 \\
0 & b & 0 & 0 \\
0 & 0 & -a & 0 \\
0 & 0 & 0 & -b
\end{array}\right): a, b \in \mathbb{R}\right\}
$$


and has the natural basis $\left\{H_{1,0}, H_{0,1}\right\}$. We define the linear functionals $\alpha$ and $\beta$ on $\mathfrak{a}$ by

$$
\alpha\left(H_{a, b}\right):=a-b, \quad \beta\left(H_{a, b}\right):=2 b .
$$

The functionals in

$$
\triangle:=\triangle^{+} \cup \triangle^{-}, \quad \triangle^{+}:=\{\alpha, \beta, \alpha+\beta, 2 \alpha+\beta\}, \quad \triangle^{-}:=\left\{-\nu: \nu \in \triangle^{+}\right\}
$$

form a so-called root system. The root system is meaningful since for any non-zero functional $\nu$ not contained in $\triangle$ the vector space

$$
\mathfrak{g}_{\nu}:=\{X \in \mathfrak{s p}(2, \mathbb{R}):[H, X]=\nu(H) X \text { for all } H \in \mathfrak{a}\}
$$

is trivial. The root spaces $\mathfrak{g}_{\nu}, \nu \in \triangle$, are one-dimensional and the linear space associated with the zero functional $\mathfrak{g}_{0}=\mathfrak{a}$ is two-dimensional. The four root vectors $X_{\nu}$ spanning the space $\mathfrak{g}_{\nu}$, $\nu \in \triangle^{+}$, are

$$
X_{\alpha}:=\left(\begin{array}{cccc}
0 & 1 & 0 & 0 \\
0 & 0 & 0 & 0 \\
0 & 0 & 0 & 0 \\
0 & 0 & -1 & 0
\end{array}\right), X_{\beta}:=\left(\begin{array}{cccc}
0 & 0 & 0 & 0 \\
0 & 0 & 0 & 1 \\
0 & 0 & 0 & 0 \\
0 & 0 & 0 & 0
\end{array}\right), X_{\alpha+\beta}:=\left(\begin{array}{cccc}
0 & 0 & 0 & 1 \\
0 & 0 & 1 & 0 \\
0 & 0 & 0 & 0 \\
0 & 0 & 0 & 0
\end{array}\right), X_{2 \alpha+\beta}:=\left(\begin{array}{cccc}
0 & 0 & 2 & 0 \\
0 & 0 & 0 & 0 \\
0 & 0 & 0 & 0 \\
0 & 0 & 0 & 0
\end{array}\right) .
$$

The root vectors $X_{-\nu}$ spanning $\mathfrak{g}_{-\nu},-\nu \in{\Delta^{-}}^{-}$, are given by the Cartan involution

$$
X_{-\nu}:=-X_{\nu}^{\mathrm{T}} .
$$

The Lie algebra $\mathfrak{s p}(2, \mathbb{R})$ has the following vector space direct sum decomposition, known as root space decomposition:

$$
\mathfrak{s p}(2, \mathbb{R})=\mathfrak{a}+\sum_{\nu \in \triangle} \mathfrak{g}_{\nu} .
$$

\begin{tabular}{|c|c|c|c|c|c|c|c|c|c|c|}
\hline$[\cdot, \cdot \cdot]$ & $X_{\alpha}$ & $X_{\beta}$ & $X_{\alpha+\beta}$ & $X_{2 \alpha+\beta}$ & $X_{-\alpha}$ & $X_{-\beta}$ & $X_{-\alpha-\beta}$ & $X_{-2 \alpha-\beta}$ & $H_{1,0}$ & $H_{0,1}$ \\
\hline $\begin{array}{l}X_{\alpha} \\
X_{\beta} \\
X_{\alpha+\beta} \\
X_{2 \alpha+\beta} \\
\end{array}$ & $\begin{array}{r}0 \\
-X_{\alpha+\beta} \\
-X_{2 \alpha+\beta} \\
0\end{array}$ & $\begin{array}{r}X_{\alpha+\beta} \\
0 \\
0 \\
0\end{array}$ & $\begin{array}{r}X_{2 \alpha+\beta} \\
0 \\
0 \\
0\end{array}$ & $\begin{array}{l}0 \\
0 \\
0 \\
0\end{array}$ & $\begin{array}{r}H_{-1,1} \\
0 \\
2 X_{\beta} \\
2 X_{\alpha+\beta} \\
\end{array}$ & $\begin{array}{r}0 \\
-H_{0,1} \\
-X_{\alpha} \\
0\end{array}$ & $\begin{array}{r}-2 X_{-\beta} \\
X_{-\alpha} \\
-H_{1,1} \\
-2 X_{\alpha} \\
\end{array}$ & $\begin{array}{r}-2 X_{-\alpha-\beta} \\
0 \\
2 X_{-\alpha} \\
-H_{4,0} \\
\end{array}$ & $\begin{array}{r}-X_{\alpha} \\
0 \\
-X_{\alpha+\beta} \\
-2 X_{2 \alpha+\beta} \\
\end{array}$ & $\begin{array}{r}X_{\alpha} \\
-2 X_{\beta} \\
-X_{\alpha+\beta} \\
0 \\
\end{array}$ \\
\hline $\begin{array}{l}X_{-\alpha} \\
X_{-\beta} \\
X_{-\alpha-\beta} \\
X_{-2 \alpha-\beta} \\
\end{array}$ & $\begin{array}{r}-H_{-1,1} \\
0 \\
2 X_{-\beta} \\
2 X_{-\alpha-\beta}\end{array}$ & $\begin{array}{r}0 \\
H_{0,1} \\
-X_{-\alpha} \\
0\end{array}$ & $\begin{array}{r}-2 X_{\beta} \\
X_{\alpha} \\
H_{1,1} \\
-2 X_{-\alpha} \\
\end{array}$ & $\begin{array}{r}-2 X_{\alpha+\beta} \\
0 \\
2 X_{\alpha} \\
H_{4,0}\end{array}$ & $\begin{array}{r}0 \\
-X_{-\alpha-\beta} \\
-X_{-2 \alpha-\beta} \\
0\end{array}$ & $\begin{array}{r}X_{-\alpha-\beta} \\
0 \\
0 \\
0\end{array}$ & $\begin{array}{r}X_{-2 \alpha-\beta} \\
0 \\
0 \\
0\end{array}$ & $\begin{array}{l}0 \\
0 \\
0 \\
0\end{array}$ & $\begin{array}{r}X_{-\alpha} \\
0 \\
X_{-\alpha-\beta} \\
2 X_{-2 \alpha-\beta} \\
\end{array}$ & $\begin{array}{r}-X_{-\alpha} \\
2 X_{-\beta} \\
X_{-\alpha-\beta} \\
0\end{array}$ \\
\hline $\begin{array}{l}H_{1,0} \\
H_{0,1}\end{array}$ & $\begin{array}{r}X_{\alpha} \\
-X_{\alpha}\end{array}$ & $\begin{array}{r}0 \\
2 X_{\beta}\end{array}$ & $\begin{array}{l}X_{\alpha+\beta} \\
X_{\alpha+\beta}\end{array}$ & $\begin{array}{r}2 X_{2 \alpha+\beta} \\
0\end{array}$ & $\begin{array}{r}-X_{-\alpha} \\
X_{-\alpha}\end{array}$ & $\begin{array}{r}0 \\
-2 X_{-\beta}\end{array}$ & $\begin{array}{l}-X_{-\alpha-\beta} \\
-X_{-\alpha-\beta}\end{array}$ & $\begin{array}{r}-2 X_{-2 \alpha-\beta} \\
0\end{array}$ & $\begin{array}{l}0 \\
0\end{array}$ & $\begin{array}{l}0 \\
0\end{array}$ \\
\hline
\end{tabular}

To show our embedding result we will label Hamiltonians with respect to the basis

$$
\begin{aligned}
\mathcal{B} & =\left\{X_{\alpha}, X_{\beta}, X_{\alpha+\beta}, X_{2 \alpha+\beta}, X_{-\alpha}, X_{-\beta}, X_{-\alpha-\beta}, X_{-2 \alpha-\beta}, H_{1,0}, H_{0,1}\right\} \\
& =\left\{B_{k}: k=1, \ldots, 10\right\}
\end{aligned}
$$

where the enumeration is with respect to the above ordering of the elements. The following table contains the commutator rules of the basis elements:

Table 1: Commutator relations $\left[B_{i}, B_{j}\right]$ for $B_{i}, B_{j} \in \mathcal{B}, i, j=1, \ldots, 10$. 
Canonical normal forms. Next we give the complete list of canonical normal forms to which we can reduce real $4 \times 4$ Hamiltonians by means of real symplectic conjugations, i.e., by applying $\operatorname{Ad}(B) X:=B X B^{-1}$ with $B \in \operatorname{Sp}(2, \mathbb{R})$. For arbitrary space dimensions and symplectic spaces over any field the result is due to Williamson 33. For real Hamiltonians the characterization can be found in a synthetic form in [1, Appendix 6] which we briefly recall for $\mathfrak{s p}(2, \mathbb{R})$ below.

The canonical normal forms are closely related to the Jordan normal forms of Hamiltonians. The eigenvalues of Hamiltonians are of four types, namely (i) real pairs $(+a,-a), a>0$, (ii) purely imaginary pairs $(+b i,-b i), b>0$, (iii) quadruples $( \pm a \pm i b), a>0, b>0$, and (iv) zeros. The Jordan blocks for the two members of a pair have the same structure, and there is an even number of blocks of odd order with zero eigenvalue.

In Arnol'd's book [1] the canonical normal forms are nicely determined by the help of a quadratic form (Hamiltonian function). To this end, note that any $X \in \mathfrak{s p}(2, \mathbb{R})$ is related to a symmetric matrix $A \in \operatorname{Sym}(4, \mathbb{R})$ by $J X=A$. Now, $A \in \operatorname{Sym}(4, \mathbb{R})$ and hence $X=-J A$ is completely determined by the quadratic form

$$
H_{A}(x):=\frac{1}{2}\langle A x, x\rangle .
$$

Using the notation $x:=\left(p_{1}, \ldots, p_{k}, q_{1}, \ldots, q_{k}\right)^{\mathrm{T}}, k \in\{1,2\}$, the list of canonical normal forms for the irreducible cases and their relation to their Jordan normal forms read as follows (order as in [1]):

(A) If $X \in \mathfrak{s p}(1, \mathbb{R})$ has a pair of Jordan blocks of order one with real eigenvalues $\pm a, a \geq 0$, then it has the normal form

$$
H_{A}\left(p_{1}, q_{1}\right)=-a p_{1} q_{1} \quad \text { and } \quad-J A=\left(\begin{array}{cc}
a & 0 \\
0 & -a
\end{array}\right) .
$$

(B) If $X \in \mathfrak{s p}(2, \mathbb{R})$ has Jordan blocks of order two with real eigenvalues $\pm a, a \geq 0$, then

$$
H_{A}\left(p_{1}, p_{2}, q_{1}, q_{2}\right)=-a\left(p_{1} q_{1}+p_{2} q_{2}\right)+p_{1} q_{2} \quad \text { and } \quad-J A=D_{2}=\left(\begin{array}{cccc}
a & 0 & 0 & 0 \\
-1 & a & 0 & 0 \\
0 & 0 & -a & 1 \\
0 & 0 & 0 & -a
\end{array}\right) \text {. }
$$

(C) If $X \in \mathfrak{s p}(2, \mathbb{R})$ has a quadruple of Jordan blocks of order one with complex eigenvalues $\pm a \pm i b, a, b>0$, then

$$
H_{A}\left(p_{1}, p_{2}, q_{1}, q_{2}\right)=-a\left(p_{1} q_{1}+p_{2} q_{2}\right)+b\left(p_{1} q_{2}-p_{2} q_{1}\right) \text { and }-J A=D_{3}=\left(\begin{array}{cccc}
a & b & 0 & 0 \\
-b & a & 0 & 0 \\
0 & 0 & -a & b \\
0 & 0 & -b & -a
\end{array}\right) \text {. }
$$

(D) If $X \in \mathfrak{s p}(2, \mathbb{R})$ has a single Jordan block of order four with eigenvalue zero, then, for $\varepsilon= \pm 1$

$$
H_{A}\left(p_{1}, p_{2}, q_{1}, q_{2}\right)=\frac{\varepsilon}{2}\left(p_{1}^{2}-2 q_{1} q_{2}\right)-p_{1} q_{2} \quad \text { and } \quad-J A=D_{4}=\left(\begin{array}{cccc}
0 & 0 & 0 & \varepsilon \\
1 & 0 & \varepsilon & 0 \\
\varepsilon & 0 & 0 & -1 \\
0 & 0 & 0 & 0
\end{array}\right) .
$$


(E) If $X \in \mathfrak{s p}(1, \mathbb{R})$ has a pair of Jordan blocks of order one with purely imaginary eigenvalues $\pm i b, b>0$, then, for $\varepsilon= \pm 1$,

$$
H_{A}\left(p_{1}, q_{1}\right)=-\frac{\varepsilon}{2}\left(b^{2} p_{1}^{2}+q_{1}^{2}\right) \quad \text { and } \quad-J A=\left(\begin{array}{cc}
0 & \varepsilon \\
-\varepsilon b^{2} & 0
\end{array}\right) .
$$

If $X \in \mathfrak{s p}(1, \mathbb{R})$ has a single Jordan block of order two with eigenvalue zero, then it has the canonical normal $H_{A}\left(p_{1}, q_{1}\right)=-\frac{\varepsilon}{2} q_{1}^{2}$, which coincides with the above form for $b=0$.

(F) If $X \in \mathfrak{s p}(2, \mathbb{R})$ has a pair of Jordan blocks of order two with purely imaginary eigenvalues $\pm i b, b>0$, then, for $\varepsilon= \pm 1$,

$$
H_{A}\left(p_{1}, p_{2}, q_{1}, q_{2}\right)=-\frac{\varepsilon}{2}\left(\frac{1}{b^{2}} q_{1}^{2}+q_{2}^{2}\right)-b^{2} p_{1} q_{2}+p_{2} q_{1} \text { and }-J A=D_{7}=\left(\begin{array}{cccc}
0 & -1 & \frac{\varepsilon}{b^{2}} & 0 \\
b^{2} & 0 & 0 & \varepsilon \\
0 & 0 & 0 & -b^{2} \\
0 & 0 & 1 & 0
\end{array}\right) .
$$

Combining the irreducible $2 \times 2$ cases $(\mathrm{A})$ and $(\mathrm{E})$ we obtain the remaining three canonical normal forms. We will denote by $(\mathrm{X}) \oplus(\mathrm{Y})$ the canonical form that corresponds to the direct sum of the quadratic forms. We then multiply on the left by $-J$ and obtain the corresponding matrix in $\mathfrak{s p}(2, \mathbb{R})$. We obtain three further cases, namely:

for $(\mathrm{A}) \oplus(\mathrm{A})$ :

$$
D_{1}=\left(\begin{array}{cccc}
a_{1} & 0 & 0 & 0 \\
0 & a_{2} & 0 & 0 \\
0 & 0 & -a_{1} & 0 \\
0 & 0 & 0 & -a_{2}
\end{array}\right), \quad a_{1} \geq a_{2} \geq 0
$$

for $(\mathrm{E}) \oplus(\mathrm{A})$ :

$$
D_{5}=\left(\begin{array}{cccc}
0 & 0 & \varepsilon & 0 \\
0 & a & 0 & 0 \\
-b^{2} \varepsilon & 0 & 0 & 0 \\
0 & 0 & 0 & -a
\end{array}\right), \quad a \geq 0, b \geq 0, \varepsilon= \pm 1
$$

and for $(\mathrm{E}) \oplus(\mathrm{E})$ :

$$
D_{6}=\left(\begin{array}{cccc}
0 & 0 & \varepsilon & 0 \\
0 & 0 & 0 & \eta \\
-b_{1}^{2} \varepsilon & 0 & 0 & 0 \\
0 & -b_{2}^{2} \eta & 0 & 0
\end{array}\right), \quad b_{1} \geq b_{2} \geq 0,(\varepsilon, \eta) \in\{(1,1),(1,-1),(-1,-1)\}
$$

We summarize our specifications of the results in [1, 33] for $d=2$ :

Corollary 4.1. For any $X \in \mathfrak{s p}(2, \mathbb{R})$, there exists $B \in \operatorname{Sp}(2, \mathbb{R})$ such that $\operatorname{Ad}(B) X \in \mathcal{N}$, where $\mathcal{N}:=\left\{D_{k}: k=1, \ldots, 7\right\}$.

\subsection{Embedding result}

To prove Theorem 3.4, we first observe that $\mathbb{S}^{+}$is simply connected so that we can pass to its Lie algebra denoted by $\mathfrak{h}_{e}^{+}$, see also Lemma 2.1 . Let $g_{*}^{+}, \kappa_{*}^{+}$and $\left(R_{-1}\right)_{*}$ be the tangent maps 
corresponding to $g^{+}, \kappa^{+}$and $R_{-1}$ in Theorem 3.4. Note that $g_{*}^{+}, \kappa_{*}^{+}$are Lie algebra embeddings of $\mathfrak{h}_{e}^{+}$into the $\mathfrak{s p}(2, \mathbb{R})$, whereas $\left(R_{-1}\right)_{*}$ is a Lie algebra isomorphism of $\mathfrak{h}_{e}^{+}$. We have to prove that there exists $B \in \operatorname{Sp}(2, \mathbb{R})$ and a Lie algebra isomorphism $\Phi: \mathfrak{h}_{e}^{+} \rightarrow \mathfrak{h}_{e}^{+}$satisfying

$$
\left(R_{-1}\right)_{*} \Phi=\Phi\left(R_{-1}\right)_{*}
$$

such that

$$
g_{*}^{+}=B\left(\kappa_{*}^{+} \Phi\right) B^{-1} \text {. }
$$

The image of $\mathfrak{h}_{e}^{+}$under $\kappa_{*}^{+}$is the Lie algebra of $\operatorname{TDS}(2)$ and, with slight abuse of notation, we identify it with $\mathfrak{h}_{e}^{+}$. By taking the derivative of the following four one-parameters subgroups,

$$
\eta \mapsto \kappa^{+}(\exp (2 \eta), 0,0,0), \eta \mapsto \kappa^{+}(1, \eta, 0,0), \eta \mapsto \kappa^{+}(1,0,2 \eta, 0), \eta \mapsto \kappa^{+}(1,0,0,2 \eta),
$$

we get a basis of $\mathfrak{h}_{e}^{+}$, namely

$$
D^{+}=-H_{1,0}+(1-2 \gamma) H_{0,1} \quad P^{+}=X_{-\alpha}, \quad Q^{+}=-X_{-\alpha-\beta}, \quad T^{+}=-X_{-2 \alpha-\beta} .
$$

By Table 1, the non-zero brackets of these generators are

$$
\left[D^{+}, P^{+}\right]=2(1-\gamma) P^{+}, \quad\left[D^{+}, Q^{+}\right]=2 \gamma Q^{+}, \quad\left[P^{+}, Q^{+}\right]=T^{+}, \quad\left[D^{+}, T^{+}\right]=2 T^{+} .
$$

The action of the Lie algebra isomorphism $\left(R_{-1}\right)_{*}$ is given by

$$
\left(R_{-1}\right)_{*} D^{+}=D^{+}, \quad\left(R_{-1}\right)_{*} P^{+}=P^{+}, \quad\left(R_{-1}\right)_{*} Q^{+}=-Q^{+}, \quad\left(R_{-1}\right)_{*} T^{+}=-T^{+} .
$$

Observe that, for any fixed $u, z \in \mathbb{R}$ with $u z \neq 0$, the linear map $\Phi: \mathfrak{h}_{e}^{+} \rightarrow \mathfrak{h}_{e}^{+}$defined by

$$
\Phi D^{+}:=D^{+}, \quad \Phi P^{+}:=u P^{+}, \quad \Phi Q^{+}:=z Q^{+}, \quad \Phi T^{+}:=u z T^{+}
$$

is a Lie algebra isomorphism satisfying (21).

An arbitrary Lie algebra embedding $g_{*}^{+}: \mathfrak{h}_{e}^{+} \rightarrow \mathfrak{s p}(2, \mathbb{R})$ is in one-to-one correspondence with four linearly independent generators $D, P, Q, T$ of $\mathfrak{s p}(2, \mathbb{R})$ whose Lie brackets are given by

$$
[D, P]=2(1-\gamma) P, \quad[D, Q]=2 \gamma Q, \quad[P, Q]=T, \quad[D, T]=2 T, \quad[P, T]=[Q, T]=0
$$

where $D, P, Q, T$ are the images of $D^{+}, P^{+}, Q^{+}, T^{+}$and hence determine $g_{*}^{+}$. Then, by (22) , it remains to prove the following theorem.

Theorem 4.2. For $\gamma \in(0,1) \backslash\left\{\frac{1}{3}, \frac{2}{3}\right\}$, let $D^{+}, P^{+}, Q^{+}, T^{+}$be given by (23). Then, for any fixed generators $D, P, Q, T \in \mathfrak{s p}(2, \mathbb{R})$ fulfilling (26), there exists $B \in \operatorname{Sp}(2, \mathbb{R})$ and a Lie algebra isomorphism $\Phi: \mathfrak{h}_{e}^{+} \rightarrow \mathfrak{h}_{e}^{+}$satisfying (21) so that

$$
\operatorname{Ad}(B) \Phi D^{+}=D, \quad \operatorname{Ad}(B) \Phi P^{+}=P, \quad \operatorname{Ad}(B) \Phi Q^{+}=Q, \quad \operatorname{Ad}(B) \Phi T^{+}=T .
$$

Note that, the choice of a map $\Phi$ as in (25) allows to change $P$ and $Q$ up to a multiplicative constant.

Proof. First we obtain by straightforward computation 
(i) for $0<\gamma \leq \frac{1}{2}$, $\Phi$ in (25) with $u=z=-1$ and $B:=\left(\begin{array}{cccc}0 & 0 & -1 & 0 \\ 0 & 1 & 0 & 0 \\ 1 & 0 & 0 & 0 \\ 0 & 0 & 0 & 1\end{array}\right) \in \operatorname{Sp}(2, \mathbb{R})$ that

$$
\begin{array}{lll}
\operatorname{Ad}(B) \Phi D^{+}=H_{1,0}+(1-2 \gamma) H_{0,1}, & & \operatorname{Ad}(B) \Phi P^{+}=X_{\alpha+\beta}, \\
\operatorname{Ad}(B) \Phi Q^{+}=X_{\alpha}, & \operatorname{Ad}(B) \Phi T^{+}=-X_{2 \alpha+\beta} .
\end{array}
$$

(ii) for $\frac{1}{2} \leq \gamma<1, \Phi$ in (25) with $u=1, z=-1$ and $B:=-J$ that

$$
\begin{aligned}
& \operatorname{Ad}(B) \Phi D^{+}=H_{1,0}-(1-2 \gamma) H_{0,1}, \quad \operatorname{Ad}(B) \Phi P^{+}=X_{\alpha}, \\
& \operatorname{Ad}(B) \Phi Q^{+}=X_{\alpha+\beta}, \quad \operatorname{Ad}(B) \Phi T^{+}=X_{2 \alpha+\beta} .
\end{aligned}
$$

We show that, up to conjugation and change of sign in the definition of $P$ and $Q$, the matrices in (27) and (28) are the unique ones which fulfill (26).

Let $D, P, Q, T \in \mathfrak{s p}(2, \mathbb{R})$ be arbitrarily fixed, linearly independent matrices with property (26). By Corollary 4.1, up to conjugation, $D$ is one of the canonical forms in $\mathcal{N}$. For each $D \in \mathcal{N}$ we have to find $P, Q \in \mathfrak{s p}(2, \mathbb{R})$ fulfilling in particular $[D, P]=2(1-\gamma) P$ and $[D, Q]=2 \gamma Q$. For this purpose we consider for all $\gamma \in(0,1)$ the pairs of vector spaces

$$
V_{\Gamma}:=\{X \in \mathfrak{s p}(2, \mathbb{R}):[D, X]=\Gamma X\}, \quad \Gamma \in\{2(1-\gamma), 2 \gamma\},
$$

which coincide in the case $\gamma=\frac{1}{2}$. We compute the Lie brackets of every $D \in \mathcal{N}$ with the basis elements in (20), i.e., we use Table 1 to find $\left[D, B_{k}\right]=\sum_{j=1}^{10} d_{k j} B_{j}, k=1, \ldots, 10$. Then we obtain for any $X:=\sum_{k=1}^{10} x_{k} B_{k} \in \mathfrak{s p}(2, \mathbb{R})$ that

$$
\begin{aligned}
{[D, X]-\Gamma X } & =\sum_{k=1}^{10} x_{k}\left[D, B_{k}\right]-\Gamma \sum_{j=1}^{10} x_{j} B_{j} \\
& =\sum_{k=1}^{10} \sum_{j=1}^{10} x_{k} d_{k j} B_{j}-\Gamma \sum_{j=1}^{10} x_{j} B_{j} \\
& =\sum_{j=1}^{10}\left(\sum_{k=1}^{10}\left(d_{k j}-\Gamma \delta_{k j}\right) x_{k}\right) B_{j}
\end{aligned}
$$

where $\delta_{k j}=1$ for $k=j$ and $\delta_{k j}=0$ otherwise (Kronecker delta). Hence $[D, X]=\Gamma X$ is equivalent to $M_{\Gamma} x=0$, where $x:=\left(x_{k}\right)_{k=1}^{10}$ and

$$
M_{\Gamma}:=\left(d_{k j}-\Gamma \delta_{k j}\right)_{j, k=1}^{10} .
$$

To have non-trivial linearly independent solutions $X$ (for $P$ and $Q$ ) we need that $M_{\Gamma}$ has rank $\leq 9$ for each $\Gamma \in\{2(1-\gamma), 2 \gamma\}$ if $\gamma \neq \frac{1}{2}$ and rank $\leq 8$ if $\gamma=\frac{1}{2}$. For the seven matrices $D \in \mathcal{N}$ the brackets $\left[D, B_{k}\right], k=1, \ldots, 10$, the corresponding matrices $M_{\Gamma}$ and their determinants are listed in the appendix. Using these computations we have the following cases:

1. For $D \in\left\{D_{4}, D_{6}, D_{7}\right\}$ we see immediately that det $M_{\Gamma} \neq 0$ for $\Gamma \in\{2(1-\gamma), 2 \gamma\}, \gamma \in(0,1)$ so that the matrices have full rank.

2. For $D \in\left\{D_{2}, D_{3}\right\}$ only $2 a=2(1-\gamma)=2 \gamma$ leads to det $M_{\Gamma}=0, \Gamma \in\{2(1-\gamma), 2 \gamma\}$. This implies $\gamma=\frac{1}{2}$ and $M_{2 \gamma}=M_{2(1-\gamma)}=M_{1}$. But $M_{1}$ has rank 9 in both cases $D \in\left\{D_{2}, D_{3}\right\}$. 
3. For $D=D_{5}$ we obtain $\operatorname{det} M_{\Gamma}=0$ for $\Gamma \in\{2(1-\gamma), 2 \gamma\}$ in the following cases:

$3.12 a=2(1-\gamma)=2 \gamma$ or $a=2(1-\gamma)=2 \gamma$ and $b=0$, which implies $\gamma=\frac{1}{2}$. However, as in the previous case, $M_{1}$ has rank 9 .

3.2 For $b=0$ : $2 a=2(1-\gamma)$ and $a=2 \gamma$ or $2 a=2 \gamma$ and $a=2(1-\gamma)$ which is only possible if $\gamma=\frac{1}{3}$ or $\gamma=\frac{2}{3}$. But for these cases the second or third column of $M_{\Gamma}$ is zero so that $P$ would be a multiple of $X_{\beta}$ and $Q$ a multiple of $X_{\alpha+\beta}$ (or vice versa) but these basis elements commute.

4. Finally, for $D=D_{1}$, the matrix $M_{\Gamma}$ is a diagonal matrix with diagonal entries $\left(a_{1}-a_{2}-\Gamma, 2 a_{2}-\Gamma, a_{1}+a_{2}-\Gamma, 2 a_{1}-\Gamma, a_{2}-a_{1}-\Gamma,-2 a_{2}-\Gamma,-a_{2}-a_{1}-\Gamma,-2 a_{1}-\Gamma,-\Gamma,-\Gamma\right)$. Since $a_{1} \geq a_{2} \geq 0$ and $\Gamma>0$, the last six elements are less than zero so that $M_{\Gamma} x=0$

\begin{tabular}{l|r|rrr}
\hline$[\cdot, \cdot]$ & $X_{\alpha}$ & $X_{\beta}$ & $X_{\alpha+\beta}$ & $X_{2 \alpha+\beta}$ \\
\hline$X_{\alpha}$ & 0 & $X_{\alpha+\beta}$ & $X_{2 \alpha+\beta}$ & 0 \\
\hline$X_{\beta}$ & $-X_{\alpha+\beta}$ & 0 & 0 & 0 \\
$X_{\alpha+\beta}$ & $-X_{2 \alpha+\beta}$ & 0 & 0 & 0 \\
$X_{2 \alpha+\beta}$ & 0 & 0 & 0 & 0 \\
\hline
\end{tabular}

Table 2: Commutator relations $\left[B_{i}, B_{j}\right]$ for $B_{i}, B_{j} \in \mathcal{B}, i, j=1, \ldots, 4$.

implies $x_{5}=x_{6}=\ldots=x_{10}=0$. Hence any solution $X$ is a linear combination of at most the first four basis elements. To obtain solutions $X$ for $P$ and $Q$ such that $[P, Q]=T \neq 0$ we see from Table 2 that at least one solution $X$ must be a nontrivial combination with $B_{1}=X_{\alpha}$, i.e., $x_{1} \neq 0$. Consequently, we need $a_{1}-a_{2}-\Gamma=0$ for one $\Gamma \in\{2(1-\gamma), 2 \gamma\}$.

Let $\gamma \neq \frac{1}{2}$. Then, for the other choice of $\Gamma$, another diagonal element has to be zero. Table 3 shows the corresponding six cases. Note that by setting the first and another diagonal element to zero, the values $a_{1}$ and $a_{2}$ are uniquely determined by $\gamma$.

The pairs $\left(a_{1}, a_{2}\right) \in\{(1-\gamma, 1-3 \gamma),(\gamma, 3 \gamma-2)\}$ lead to a solution $X$ which is a multiple of $X_{2 \alpha+\beta}$ and consequently commutes with all four basis elements, see Table 2 . This contradicts the requirement $[P, Q]=T \neq 0$.

For $\left(a_{1}, a_{2}\right) \in\{(\gamma+1,1-\gamma),(2-\gamma, \gamma)\}$ the solutions $X$ (for $P$ and $Q$ ) are multiples of $X_{\alpha}$ and $X_{\beta}$, so that by Table 2, the matrix $T$ becomes a multiple of $X_{\alpha+\beta}$. But this $T$ cannot commute with $P$ and $Q$ as required by (24).

For $\left(a_{1}, a_{2}\right)=(1,1-2 \gamma)$ with $\gamma<\frac{1}{2}, \gamma \neq \frac{1}{3}$ we obtain (up to multiplication with scalars) the setting (27), and for $\left(a_{1}, a_{2}\right)=(1,2 \gamma-1)$ with $\gamma>\frac{1}{2}, \gamma \neq \frac{2}{3}$ the result (28). For $\gamma \in\left\{\frac{1}{3}, \frac{2}{3}\right\}$ see Remark 4.3 ,

Let $\gamma=\frac{1}{2}$ which implies $\Gamma=1$. Then, regarding that $x_{1} \neq 0$, and consequently $a_{1}-a_{2}-1=$ 0, i.e., $a_{2}=a_{1}-1$, the first four diagonal elements of $M_{1}$ must read as

$$
\left(0,2 a_{1}-3,2 a_{1}-2,2 a_{1}-1\right) .
$$

The matrix $M_{1}$ must have rank $\leq 8$ and $a_{2} \geq 0$. This is only possible if $\left(a_{1}, a_{2}\right) \in$ $\left\{\left(\frac{3}{2}, \frac{1}{2}\right),(1,0)\right\}$. For $\left(a_{1}, a_{2}\right)=\left(\frac{3}{2}, \frac{1}{2}\right)$ the solutions $X$ for $P$ and $Q$ are multiples of $X_{\alpha}$ 


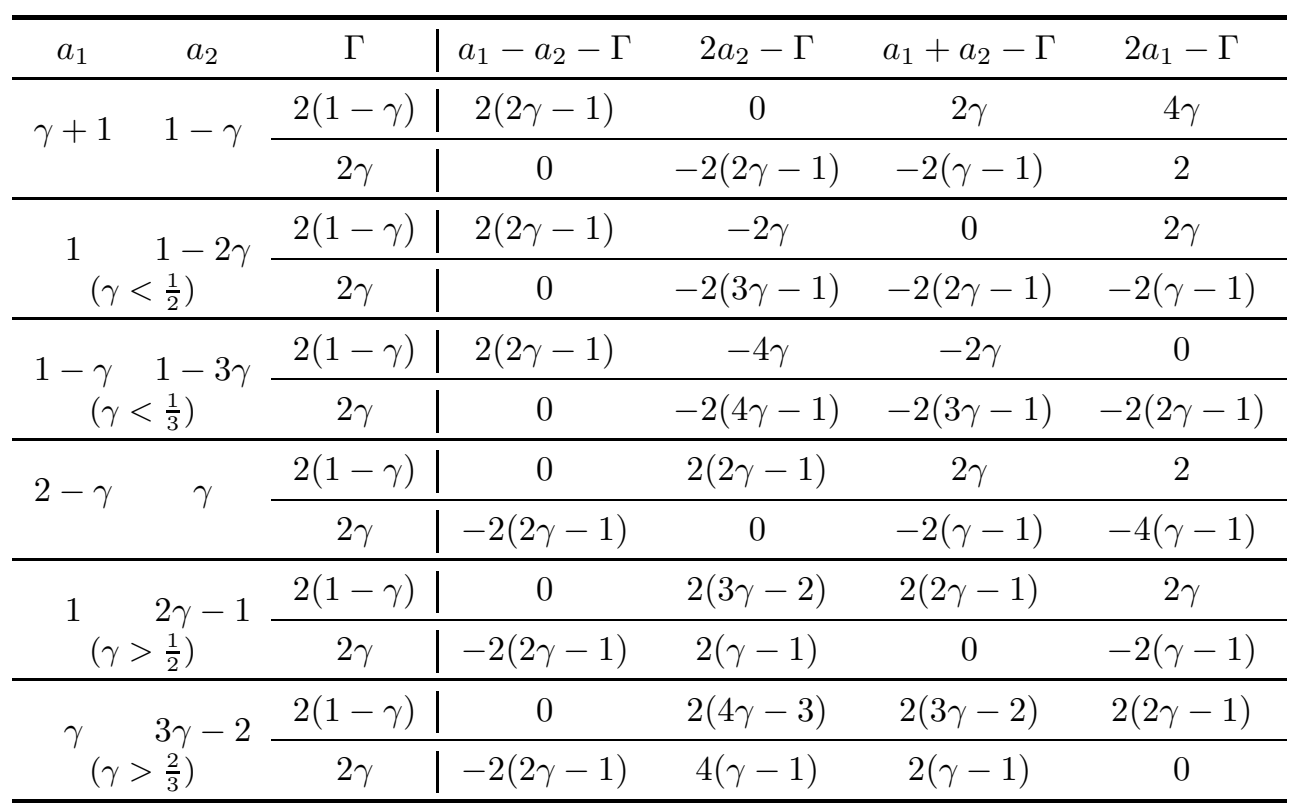

Table 3: Possible solutions for $a_{1}$ and $a_{2}$ such that $a_{1}-a_{2}-\Gamma=0$ (the first entry of $M_{\Gamma}$ ).

and $X_{\beta}$. But then $T=[P, Q]$ is a multiple of $X_{\alpha+\beta}$ which does not commute with both $X_{\alpha}$ and $X_{\beta}$ as required by (24).

For $\left(a_{1}, a_{2}\right)=(1,0)$ we obtain the solution

$$
D=H_{1,0} \quad P=u X_{\alpha}+v X_{\alpha+\beta}, \quad Q=w X_{\alpha}+z X_{\alpha+\beta}, \quad T=(u z-v w) X_{2 \alpha+\beta},
$$

where $u z-w v \neq 0$ must be fulfilled. Possibly changing $P$ into $-P$, we can assume that $u z-w v>0$. Now straightforward computation shows that

$$
C:=\frac{1}{m}\left(\begin{array}{cccc}
m^{2} & 0 & 0 & 0 \\
0 & z & 0 & -v \\
0 & 0 & 1 & 0 \\
0 & -w & 0 & u
\end{array}\right), \quad m:=\sqrt{u z-v w}
$$

is a symplectic matrix which fulfills

$$
\operatorname{Ad}(C) H_{1,0}=H_{1,0}, \quad \operatorname{Ad}(C) X_{\alpha}=u X_{\alpha}+v X_{\alpha+\beta}, \quad \operatorname{Ad}(C) X_{\alpha+\beta}=w X_{\alpha}+z X_{\alpha+\beta} .
$$

This finishes the proof.

Remark 4.3. For $\gamma=\frac{1}{3}$ (and $\gamma=\frac{2}{3}$ ) there are 'non-standard' embeddings $\widetilde{\kappa}$ of $\mathbb{S}^{+}$into $\operatorname{Sp}(2, \mathbb{R})$, which are not conjugated with (10) unless $w=0$. At the Lie algebra level, $\widetilde{\kappa}_{*}: \mathfrak{h}_{e}^{+} \rightarrow$ $\mathfrak{s p}(2, \mathbb{R})$ acts as

$$
\widetilde{\kappa}_{*} D^{+}=H_{1,0}+\frac{1}{3} H_{0,1} \quad \widetilde{\kappa}_{*} P^{+}=u X_{\alpha+\beta} \quad \widetilde{\kappa}_{*} Q^{+}=v X_{\alpha}+w X_{\beta} \quad \widetilde{\kappa}_{*} Z^{+}=-u v X_{2 \alpha+\beta} .
$$

However, it is easy to check that for such embeddings there does not exist a symplectic matrix A satisfying (15). For further explanations we refer to [24]. 


\section{Coorbit spaces for equivalent representations}

In this section we show the relation between the coorbit spaces of isomorphic groups with equivalent representations and apply it to our setting. We briefly introduce the general coorbit space theory and describe how isomorphic groups with equivalent representations lead to isomorphic scales of coorbit spaces. Then we specify the results for our connected shearlet and shearlet Toeplitz groups and their isomorphic subgroups of the symplectic group. Since the latter ones are equipped with a metaplectic representation this leads finally to metaplectic coorbit spaces.

\subsection{Coorbit spaces}

Let $G$ be a locally compact group with left Haar measure $d \mu$. A unitary representation of $G$ on a Hilbert space $\mathcal{H}$ is a homomorphism $\pi$ from $G$ into the group $\mathcal{U}(\mathcal{H})$ of unitary operators on $\mathcal{H}$ that is continuous with respect to the strong operator topology, see [17. A representation is called irreducible if there does not exist a nontrivial $\pi$-invariant subspace of $\mathcal{H}$. A unitary, irreducible representation $\pi$ fulfilling

$$
\int_{G}|\langle\psi, \pi(g) \psi\rangle|^{2} d \mu(g)<\infty
$$

for some $\psi \in \mathcal{H}$ is called square integrable and a function fulfilling (30) admissible. Assume that there exists a square integrable representation $\pi$ of $G$. For an admissible function $\psi \in \mathcal{H}$ the mapping $V_{\psi}: \mathcal{H} \rightarrow L_{2}(G)$ with $V_{\psi}(f)(g):=\langle f, \pi(g) \psi\rangle$ is known as voice transform of $f$ (with respect to $\psi$ ). The admissibility condition (30) is important since it yields to a resolution of the identity that allows the reconstruction of a function $f \in \mathcal{H}$ from its voice transform $(\langle f, \pi(g) \psi\rangle)_{g \in G}$. Using the voice transform we can reformulate the admissibility condition (30) as $V_{\psi}(\psi) \in L_{2}(G)$.

For a general real-valued weight w and $1 \leq p \leq \infty$ we define the weighted $L_{p}$ space on $G$ as

$$
L_{p, \mathrm{w}}(G):=\left\{F \text { measurable }: F \mathrm{w} \in L_{p}(G)\right\} .
$$

Further we will need the weighted sequence spaces

$$
\ell_{p, \mathrm{w}}:=\left\{\left(c_{i}\right)_{i \in \mathcal{I}}:\left(c_{i} \mathrm{w}_{\mathrm{i}}\right)_{i \in \mathcal{I}} \in \ell_{p}\right\} .
$$

The voice transform can be extended from the Hilbert space $\mathcal{H}$ to weighted Banach spaces of distributions using coorbit space theory. This theory was developed by Feichtinger and Gröchenig in a series of papers [14, 15, 16, 18, 20] and we collect the basic ideas in the following. Let now $w$ be a real-valued, continuous and submultiplicative weight on $G$, i.e., $w(g h) \leq$ $w(g) w(h)$ for all $g, h \in G$ fulfilling in addition the conditions stated in [18, Section 2.2]. We assume that the so-called set of analyzing vectors

$$
\mathcal{A}_{w}:=\left\{\psi \in \mathcal{H}: V_{\psi}(\psi) \in L_{1, w}(G)\right\} .
$$

is nonempty and fix a nontrivial function $\psi \in \mathcal{A}_{w}$. We can define a set of test functions and equip it with a norm such that it becomes a Banach space by

$$
\mathcal{H}_{1, w}:=\left\{f \in \mathcal{H}: V_{\psi}(f) \in L_{1, w}(G)\right\}, \quad\|f\|_{\mathcal{H}_{1, w}}:=\left\|V_{\psi}(f)\right\|_{L_{1, w}(G)} .
$$


Its anti-dual space, i.e., the space of all continuous conjugate-linear functionals on $\mathcal{H}_{1, w}$, also called space of distributions, is denoted by $\mathcal{H}_{1, w}^{\sim}$. The definitions of $\mathcal{H}_{1, w}$ and $\mathcal{H}_{1, w}^{\sim}$ are independent of the choice of the analyzing vector $\psi \in \mathcal{A}_{w}$, see [14, Lemma 4.2], in particular $\mathcal{H}_{1, w}=\mathcal{A}_{w}$ as sets. The spaces $\mathcal{H}_{1, w}$ and $\mathcal{H}_{1, w}^{\sim}$ are $\pi$-invariant Banach spaces with continuous embeddings $\mathcal{H}_{1, w} \hookrightarrow \mathcal{H} \hookrightarrow \mathcal{H}_{1, w}^{\sim}$. The inner product on $\mathcal{H} \times \mathcal{H}$ extends to a sesquilinear form on $\mathcal{H}_{1, w}^{\sim} \times \mathcal{H}_{1, w}$ : for $\psi \in \mathcal{H}_{1, w}$ and $f \in \mathcal{H}_{1, w}^{\sim}$ the extended representation coefficients

$$
V_{\psi}(f)(g):=\langle f, \pi(g) \psi\rangle_{\mathcal{H}_{1, w}^{\sim}} \times \mathcal{H}_{1, w}
$$

are well-defined and provide the desired generalization of the voice transform on $\mathcal{H}_{1, w}^{\sim}$.

Let $m$ be a $w$-moderate weight on $G$ which means that $m(x y z) \leq w(x) m(y) w(z)$ for all $x, y, z \in$ $G$. The coorbit space of $L_{p, m}(G)$ is given by

$$
\operatorname{Co}\left(L_{p, m}(G)\right):=\mathcal{H}_{p, m}:=\left\{f \in \mathcal{H}_{1, w}^{\sim}: V_{\psi}(f)=\langle f, \pi(\cdot) \psi\rangle_{\mathcal{H}_{1, w}^{\sim} \times \mathcal{H}_{1, w}} \in L_{p, m}(G)\right\}
$$

with norm $\|f\|_{\mathcal{H}_{p, m}}=\left\|\langle f, \pi(\cdot) \psi\rangle_{\mathcal{H}_{1, w}^{\sim} \times \mathcal{H}_{1, w}}\right\|_{L_{p, m}(G)}$. It is a $\pi$-invariant Banach space which does not depend on the choice of the analyzing vector $\psi \in \mathcal{A}_{w}$, see [15, Theorem 4.2]. In particular, the spaces $\mathcal{H}, \mathcal{H}_{1, w}$ and $\mathcal{H}_{1, w}^{\sim}$ can be identified with the following coorbit spaces

$$
\mathcal{H}=\operatorname{Co}\left(L_{2}(G)\right), \quad \mathcal{H}_{1, w}=\operatorname{Co}\left(L_{1, w}(G)\right), \quad \text { and } \quad \mathcal{H}_{1, w}^{\sim}=\operatorname{Co}\left(L_{\infty, \frac{1}{w}}(G)\right) .
$$

To establish atomic decompositions and Banach frames for coorbit spaces we need (i) a stronger integrability condition for the analyzing functions than (31), and (ii) a reasonable discretization of our group $G$. Concerning (i) we require that the following better subset (or set of basic atoms) is nontrivial

$$
\mathcal{B}_{w}:=\left\{\psi \in \mathcal{H}: V_{\psi}(\psi) \in \mathcal{W}^{L}\left(L_{\infty}(G), L_{1, w}(G)\right)\right\}
$$

where

$$
\mathcal{W}^{L}\left(L_{\infty}(G), L_{1, w}(G)\right):=\left\{F \in L_{\infty, \mathrm{loc}}: H_{F} \in L_{1, w}(G)\right\}
$$

and $H_{F}: G \rightarrow \mathbb{R}$ is given by $H_{F}(x):=\left\|\left(L_{x} \chi_{Q}\right) F\right\|_{\infty}=\sup _{y \in x Q}|F(y)|$, with $Q$ being a relatively compact neighborhood of the identity element $e \in G$. Then we choose $0 \neq \psi \in \mathcal{B}_{w}$. With respect to (ii) we assume that $G$ can be discretized on a so-called well-spread set. A (countable) family $X=\left\{g_{i}: i \in \mathcal{I}\right\}$ in $G$ is called well-spread if $\bigcup_{i \in \mathcal{I}} g_{i} U=G$ for some compact set $U$ with non-void interior, and if for all compact sets $K \subset G$ there exists a constant $C_{K}$ such that

$$
\sup _{j \in \mathcal{I}} \#\left\{i \in \mathcal{I}: g_{i} K \cap g_{j} K \neq \emptyset\right\} \leq C_{K} .
$$

The following theorem collects results about the existence of atomic decompositions and Banach frames from [18, 10, 7].

Theorem 5.1. Let $1 \leq p \leq \infty$ and $\psi \in \mathcal{B}_{w}, \psi \neq 0$. Then there exists a (sufficiently small) neighborhood $U \subset G$ of e such that for any well-spread set $X=\left\{g_{i}: i \in \mathcal{I}\right\}$ in $G$ the set $\left\{\pi\left(g_{i}\right) \psi: i \in \mathcal{I}\right\}$ provides an atomic decomposition and a Banach frame for $\mathcal{H}_{p, m}$.

Atomic decomposition. Every $f \in \mathcal{H}_{p, m}$ possesses an expansion

$$
f=\sum_{i \in \mathcal{I}} c_{i}(f) \pi\left(g_{i}\right) \psi
$$


where the sequence of coefficients $\left(c_{i}(f)\right)_{i \in \mathcal{I}}$ depends linearly on $f$ and satisfies

$$
\left\|\left(c_{i}(f)\right)_{i \in \mathcal{I}}\right\|_{\ell_{p, m}} \leq C\|f\|_{\mathcal{H}_{p, m}}
$$

with a constant $C$ only depending on $\psi$. Conversely, if $\left(c_{i}\right)_{i \in \mathcal{I}} \in \ell_{p, m}$, then $f=\sum_{i \in \mathcal{I}} c_{i} \pi\left(g_{i}\right) \psi$ is in $\mathcal{H}_{p, m}$ and

$$
\|f\|_{\mathcal{H}_{p, m}} \leq C^{\prime}\left\|\left(c_{i}\right)_{i \in \mathcal{I}}\right\|_{\ell_{p, m}} .
$$

Banach frames. The set $\left\{\pi\left(g_{i}\right) \psi: i \in \mathcal{I}\right\}$ is a Banach frame for $\mathcal{H}_{p, m}$ which means that there exist two constants $C_{1}, C_{2}>0$ depending only on $\psi$ such that

$$
C_{1}\|f\|_{\mathcal{H}_{p, m}} \leq\left\|\left(\left\langle f, \pi\left(g_{i}\right) \psi\right\rangle_{\mathcal{H}_{1, w} \times \mathcal{H}_{1, w}}\right)_{i \in \mathcal{I}}\right\|_{\ell_{p, m}} \leq C_{2}\|f\|_{\mathcal{H}_{p, m}}
$$

and there exists a bounded, linear reconstruction operator $\mathcal{R}$ from $\ell_{p, m}$ to $\mathcal{H}_{p, m}$ such that

$$
\mathcal{R}\left(\left(\left\langle f, \pi\left(g_{i}\right) \psi\right\rangle_{\mathcal{H}_{1, w}^{\sim}} \times \mathcal{H}_{1, w}\right)_{i \in \mathcal{I}}\right)=f .
$$

\subsection{Coorbit spaces for isomorphic groups and equivalent representations}

Let $G$ and $\widetilde{G}$ be locally compact groups with left Haar measures $d \mu$ and $d \widetilde{\mu}$, respectively, which are isomorphic with isomorphism $\iota: G \rightarrow \widetilde{G}$. Further, let $\mathcal{H}$ and $\widetilde{\mathcal{H}}$ be Hilbert spaces with isometric isomorphism $\Psi: \mathcal{H} \rightarrow \widetilde{\mathcal{H}}$. Let $\pi: G \rightarrow \mathcal{U}(\mathcal{H})$ and $\widetilde{\pi}: \widetilde{G} \rightarrow \mathcal{U}(\widetilde{\mathcal{H}})$ be unitary representations of $G$ on $\mathcal{H}$ and of $\widetilde{G}$ on $\widetilde{\mathcal{H}}$, respectively, so that for all $g \in G$ and all $f \in \mathcal{H}$

$$
\Psi(\pi(g) f)=\tilde{\pi}(\iota(g))(\Psi f) .
$$

We will refer to such $\pi$ and $\widetilde{\pi}$ as equivalent representations. Setting $\widetilde{f}:=\Psi f$, i.e., $f=\Psi^{-1} \widetilde{f}$ this can be rewritten as $\Psi\left(\pi(g) \Psi^{-1} \widetilde{f}\right)=\tilde{\pi}(\iota(g)) \tilde{f}$. The following diagram illustrates the relations.

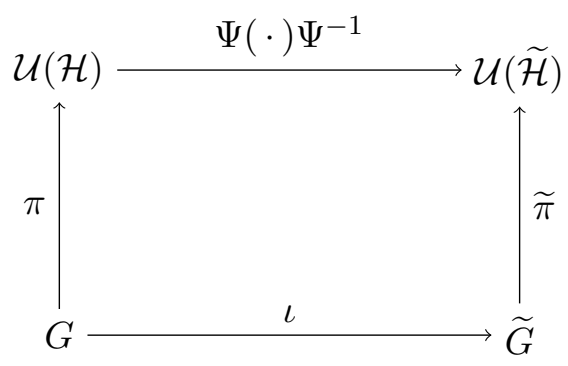

Assume that $G, \mathcal{H}$ and $\pi$ give rise to a sequence of coorbit spaces including atomic decompositions and Banach frames as described in the previous Subsection 5.1. For the corresponding weights and function spaces we use the notation from Subsection 5.1. We are interested in the relation to the coorbit spaces based on $\widetilde{G}, \widetilde{\mathcal{H}}$ and $\widetilde{\pi}$ in terms of $\iota$ and $\Psi$.

First, we define $\widetilde{w}:=w \circ \iota^{-1}$ which is clearly a real-valued, continuous, submultiplicative weight on $\widetilde{G}$ satisfying the conditions in [18, Section 2.2], since $w$ does. Let

$$
\widetilde{\mathcal{A}}_{\widetilde{w}}:=\left\{\widetilde{\psi} \in \widetilde{\mathcal{H}}:\langle\widetilde{\psi}, \widetilde{\pi}(\widetilde{g}) \widetilde{\psi}\rangle_{\widetilde{\mathcal{H}}} \in L_{1, \widetilde{w}}(\widetilde{G})\right\} .
$$


Setting $\widetilde{\psi}:=\Psi \psi$ for $\psi \in \mathcal{A}_{w}$ we obtain by (32) and since $\Psi$ is an isometry

$$
\left\langle\psi, \pi\left(\iota^{-1}(\widetilde{g})\right) \psi\right\rangle_{\mathcal{H}}=\left\langle\Psi \psi, \Psi\left(\pi\left(\iota^{-1}(\widetilde{g})\right) \psi\right)\right\rangle_{\widetilde{\mathcal{H}}}=\langle\widetilde{\psi}, \widetilde{\pi}(\widetilde{g}) \widetilde{\psi}\rangle_{\widetilde{\mathcal{H}}}
$$

Thus, $\mathcal{A}_{w}$ and $\widetilde{\mathcal{A}}_{\widetilde{w}}$ are isomorphic. For an analyzing vector $\widetilde{\psi}=\Psi \psi \in \widetilde{\mathcal{A}}_{\widetilde{w}}$ we introduce the set of test functions

$$
\widetilde{\mathcal{H}}_{1, \widetilde{w}}:=\left\{\widetilde{f} \in \mathcal{H}:\langle\widetilde{f}, \widetilde{\pi}(\widetilde{g}) \widetilde{\psi}\rangle \in L_{1, \widetilde{w}}(\widetilde{G})\right\} .
$$

Since $\|f\|_{\mathcal{H}_{1, w}}=\|\langle f, \pi(g) \psi\rangle\|_{L_{1, w}(G)}=\|\langle\Psi f, \tilde{\pi}(\iota(g)) \Psi \psi\rangle\|_{L_{1, w}(G)}$ we see with $\widetilde{g}=\iota(g)$ and $\widetilde{\psi}=\Psi \psi$ that

$$
\|f\|_{\mathcal{H}_{1, w}}=\|\langle f, \pi(g) \psi\rangle\|_{L_{1, w}(G)}=\|\langle\widetilde{f}, \widetilde{\pi}(\widetilde{g}) \widetilde{\psi}\rangle\|_{L_{1, \widetilde{w}}(\widetilde{G})}=\|\widetilde{f}\|_{\widetilde{\mathcal{H}}_{1, \widetilde{w}}} .
$$

As for the set of analyzing vectors $\Psi$ induces an isomorphism from $\mathcal{H}_{1, w}$ onto $\widetilde{\mathcal{H}}_{1, \widetilde{w}}$, where $\widetilde{f}=\Psi f$. Let $\left(\widetilde{\mathcal{H}}_{1, \widetilde{w}}\right)^{\sim}$ denote the anti-dual space of $\widetilde{\mathcal{H}}_{1, \widetilde{w}}$. This space is related to $\mathcal{H}_{1, w}^{\sim}$ by $\Psi^{*} \widetilde{f}=f$ for all $\tilde{f} \in\left(\widetilde{\mathcal{H}}_{1, \widetilde{w}}\right)^{\sim}$, where $\Psi^{*}:\left(\widetilde{\mathcal{H}}_{1, \widetilde{w}}\right)^{\sim} \rightarrow \mathcal{H}_{1, w}^{\sim}$ denotes the adjoint of the isomorphism $\Psi: \mathcal{H}_{1, w} \rightarrow \widetilde{\mathcal{H}}_{1, \widetilde{w}}$. The relations are illustrated in the following diagram.

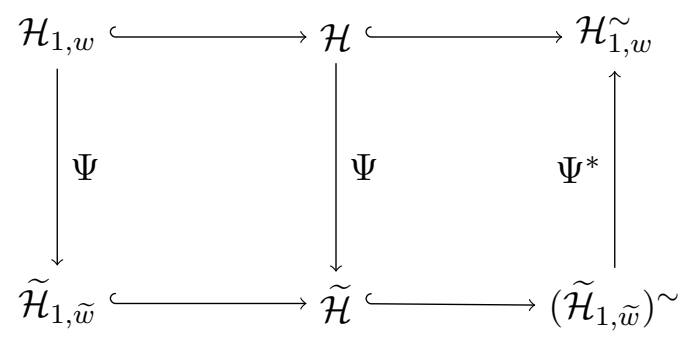

The inner product on $\widetilde{\mathcal{H}}$ extends to a dual pairing on $\left(\widetilde{\mathcal{H}}_{1, \widetilde{w}}\right)^{\sim} \times \widetilde{\mathcal{H}}_{1, \widetilde{w}}$ by

$$
\begin{aligned}
\langle\tilde{f}, \widetilde{\pi}(\widetilde{g}) \widetilde{\psi}\rangle_{\left(\widetilde{\mathcal{H}}_{1, w}\right) \sim \times \widetilde{\mathcal{H}}_{1, w}} & =\langle\tilde{f}, \Psi(\pi(g) \psi)\rangle_{\left(\widetilde{\mathcal{H}}_{1, w}\right) \sim \times \widetilde{\mathcal{H}}_{1, w}}=\left\langle\Psi^{*} \tilde{f}, \pi(g) \psi\right\rangle_{\mathcal{H}_{1, w} \times \mathcal{H}_{1, w}} \\
& =\langle f, \pi(g) \psi\rangle_{\mathcal{H}_{1, w} \times \mathcal{H}_{1, w}} .
\end{aligned}
$$

It follows that both lines of the diagram are isomorphic Gelfand triples and the extended voice transforms coincide in the sense of (33). To the $w$-moderate weight function $m$ on $G$ we associate the $\widetilde{w}$-moderate function $\widetilde{m}$ on $\widetilde{G}$ by $\widetilde{m}(\widetilde{g}):=m\left(\iota^{-1}(\widetilde{g})\right)$. Now we are ready to define the coorbit spaces of $L_{p, \widetilde{m}}(\widetilde{G})$ by

$$
\widetilde{\mathcal{H}}_{p, \widetilde{m}}:=\left\{\widetilde{f} \in\left(\widetilde{\mathcal{H}}_{1, w}\right)^{\sim}:\langle\widetilde{f}, \widetilde{\pi}(\cdot) \widetilde{\psi}\rangle_{\left(\widetilde{\mathcal{H}}_{1, w}\right) \sim \times \widetilde{\mathcal{H}}_{1, w}} \in L_{p, \widetilde{m}}(\widetilde{G})\right\}
$$

which are isomorphic to $\mathcal{H}_{p, m}$ with isomorphism $\tilde{f}=\Psi f$.

Finally, we want to analyze the relation between atomic decompositions and Banach frames of the isomorphic coorbit spaces $\mathcal{H}_{p, m}$ and $\widetilde{\mathcal{H}}_{p, \widetilde{m}}$. Clearly, the set $\widetilde{\mathcal{B}}_{\widetilde{w}}$ is just given by $\Psi\left(\mathcal{B}_{w}\right)$. Further, given a well-spread set $X:=\left\{g_{i}: i \in \mathcal{I}\right\}$ of $G$ we can check that $\widetilde{X}=\iota(X)=\left\{\widetilde{g}_{i}=\right.$ $\left.\iota\left(g_{i}\right): i \in \mathcal{I}\right\}$ is a well-spread set of $\widetilde{G}$. Then, we obtain from the atomic decomposition

$$
f=\sum_{i \in \mathcal{I}} c_{i}(f) \pi\left(g_{i}\right) \psi
$$


of $f \in \mathcal{H}_{p, m}$ and the atomic decomposition of $\tilde{f}=\Psi f$ by

$$
\widetilde{f}=\Psi f=\Psi\left(\sum_{i \in \mathcal{I}} c_{i}(f) \pi\left(g_{i}\right) \psi\right)=\sum_{i \in \mathcal{I}} c_{i}(f) \widetilde{\pi}\left(\widetilde{g}_{i}\right) \widetilde{\psi},
$$

i.e., $\widetilde{f}$ can be decomposed using the same sequence of coefficients. Concerning the Banach frame property of $\left\{\widetilde{\pi}\left(\widetilde{g}_{i}\right) \widetilde{\psi}: i \in \mathcal{I}\right\}$ it remains to deduce the reconstruction operators $\widetilde{\mathcal{R}}: \ell_{p, \widetilde{m}} \rightarrow \widetilde{\mathcal{H}}_{p, \widetilde{m}}$ given a reconstruction operator $\mathcal{R}: \ell_{p, m} \rightarrow \mathcal{H}_{p, m}$. Let the sequence of moments of $\widetilde{f}$ be given as $\left\{\left\langle\widetilde{f}, \widetilde{\pi}\left(g_{i}\right) \widetilde{\psi}\right\rangle_{\left(\widetilde{\mathcal{H}}_{1, w}\right) \sim \times \widetilde{\mathcal{H}}_{1, w}}\right\} \in \ell_{p, \widetilde{m}}$. Using $f=\Psi^{*} \widetilde{f}$ we get

$$
\left\langle\widetilde{f}, \widetilde{\pi}\left(\widetilde{g}_{i}\right) \widetilde{\psi}\right\rangle_{\left(\widetilde{\mathcal{H}}_{1, w}\right) \sim \times \widetilde{\mathcal{H}}_{1, w}}=\left\langle\widetilde{f}, \Psi\left(\pi\left(g_{i}\right) \psi\right)\right\rangle_{\left(\widetilde{\mathcal{H}}_{1, w}\right) \sim \times \widetilde{\mathcal{H}}_{1, w}}=\left\langle\Psi^{*} \widetilde{f}, \pi\left(g_{i}\right) \psi\right\rangle_{\mathcal{H}_{1, w} \times \mathcal{H}_{1, w}}
$$

and since $\mathcal{R}\left(\left\{\left\langle\Psi^{*} \widetilde{f}, \pi\left(g_{i}\right) \psi\right\rangle\right\}_{i \in \mathcal{I}}\right)=\Psi^{*} \widetilde{f}$ that $\widetilde{\mathcal{R}}=\left(\Psi^{*}\right)^{-1} \circ \mathcal{R}$.

\subsection{Application to the connected (Toeplitz) shearlet groups and their isomorphic subgroups in the symplectic group}

In this section we want to use the results from the previous section to establish coorbit spaces for the groups $\operatorname{TDS}(d, \mathbb{R})$ and $\operatorname{TDS}_{T}(d, \mathbb{R})$ using the coorbit spaces for $\mathbb{S}^{+}$and $\mathbb{S}_{T}^{+}$. First, we have to determine the latter ones.

Square integrable representations of $\mathbb{S}^{+}$and $\mathbb{S}_{T}^{+}$. The coorbit spaces for the full shearlet group $\mathbb{S}$ and $\mathcal{H}=L_{2}\left(\mathbb{R}^{d}\right)$ were introduced in [10, 11] and for the full Toeplitz shearlet group $\mathbb{S}_{T}$ in [8]. However, we cannot use these results directly since the respective representations are not irreducible if restricted to the connected groups $\mathbb{S}^{+}$and $\mathbb{S}_{T}^{+}$. Therefore, we consider instead of $L_{2}\left(\mathbb{R}^{d}\right)$ the Hilbert space

$$
L_{2}\left(\Theta_{L}\right):=\left\{f \in L_{2}\left(\mathbb{R}^{d}\right): \operatorname{supp} \hat{f} \subseteq \Theta_{L}\right\}
$$

where $\Theta_{L}$ denotes the halfspace

$$
\Theta_{L}:=\left\{\xi \in \mathbb{R}^{d}: \xi_{1} \leq 0\right\}
$$

and $\hat{f}=\mathcal{F} f$ is the Fourier transform of $f$.

To shorten the notation we write in the following $\mathbb{S}_{(T)}^{+}$to address both $\mathbb{S}^{+}$and $\mathbb{S}_{T}^{+}$. Further, we just use $A$ for the dilations in (11) and $S$ for the shears in (2) for both groups and denote by $\mu$ their left Haar measures. We define a representation $\pi: \mathbb{S}_{(T)}^{+} \rightarrow \mathcal{U}\left(L_{2}\left(\Theta_{L}\right)\right)$ by

$$
\pi(a, s, t) f(x)=f_{a, s, t}(x):=(\operatorname{det}(A))^{-\frac{1}{2}} f\left(A^{-1} S^{-1}(x-t)\right)
$$

for all $(a, s, t) \in \mathbb{S}_{(T)}^{+}$and all $f \in L_{2}\left(\Theta_{L}\right)$. The Fourier transform $\hat{f}_{a, s, t}$ of $f_{a, s, t}$ is given by

$$
\hat{f}_{a, s, t}(\omega):=\hat{\pi}(a, s, t) \hat{f}(\omega)=(\operatorname{det}(A))^{\frac{1}{2}} \hat{f}\left(A^{\mathrm{T}} S^{\mathrm{T}} \omega\right) e^{-2 \pi i\langle t, \omega\rangle} .
$$

Note that the representations $\pi$ and $\hat{\pi}$ are equivalent in the sense that

$$
\mathcal{F} \pi \mathcal{F}^{-1}=\hat{\pi}
$$


Similarly as it is done for the full shearlet and shearlet Toeplitz group in [9, 10, 11, 12, we can prove that $\pi$ is indeed a unitary representation of $\mathbb{S}_{(T)}^{+}$on $L_{2}\left(\Theta_{L}\right)$.

The following lemma shows that the unitary representation $\pi$ defined in (34) is also square integrable.

Lemma 5.2. A function $\psi \in L_{2}\left(\Theta_{L}\right)$ is admissible if and only if it fulfills the admissibility condition

$$
0<C_{\psi}:=\int_{\Theta_{L}} \frac{|\hat{\psi}(\omega)|^{2}}{\left|\omega_{1}\right|^{d}} d \omega<\infty
$$

Then, for any $f \in L_{2}\left(\Theta_{L}\right)$ the following equality holds true:

$$
\int_{\mathbb{S}_{(T)}^{+}}\left|\left\langle f, \psi_{a, s, t}\right\rangle\right|^{2} d \mu(a, s, t)=C_{\psi}\|f\|_{L_{2}\left(\Theta_{L}\right)}^{2}
$$

In particular, the unitary representation $\pi$ is irreducible and hence square integrable.

Proof. Observe that

$$
\mathcal{S} \mathcal{H}_{f}(a, s, t):=\left\langle f, \psi_{a, s, t}\right\rangle=\left\langle f, \operatorname{det}(A)^{-\frac{1}{2}} \psi\left(A^{-1} S^{-1}(\cdot-t)\right)\right\rangle=f * \psi_{a, s, 0}^{*}(t),
$$

where $\psi_{a, s, t}^{*}:=\overline{\psi_{a, s, t}(-\cdot)}$. For fixed $a$ and $s$, since $f, \psi_{a, s, 0} \in L_{2}\left(\mathbb{R}^{d}\right)$, then by standard facts $f * \psi_{a, s, 0}=\mathcal{F}^{-1}\left(\hat{f} \widehat{\psi}_{a, s, 0}^{*}\right)$ and by Plancherel's theorem we obtain

$$
\int_{\mathbb{R}^{d}}\left|f * \psi_{a, s, 0}^{*}(t)\right|^{2} d t=\int_{\widehat{\mathbb{R}}^{d}}|\hat{f}(\omega)|^{2}\left|\widehat{\psi}_{a, s, 0}^{*}(\omega)\right|^{2} d \omega
$$

where the left hand side is finite if and only if the right hand side is such. Hence, by Fubini, (35), and (38) we obtain

$$
\begin{aligned}
\int_{\mathbb{S}_{(T)}^{+}}\left|\left\langle f, \psi_{a, s, t}\right\rangle\right|^{2} \frac{d a}{a^{d+1}} d s d t & =\int_{\mathbb{S}_{(T)}^{+}}\left|f * \psi_{a, s, 0}^{*}(t)\right|^{2} d t d s \frac{d a}{a^{d+1}} \\
& =\int_{\mathbb{R}_{+}} \int_{\mathbb{R}^{d-1}} \int_{\Theta_{L}}|\hat{f}(\omega)|^{2}\left|\widehat{\psi^{*}}{ }_{a, s, 0}(\omega)\right|^{2} d \omega d s \frac{d a}{a^{d+1}} \\
& =\int_{\mathbb{R}_{+}} \int_{\mathbb{R}^{d-1}} \int_{\Theta_{L}}|\hat{f}(\omega)|^{2} \frac{\operatorname{det}(A)}{a^{d+1}}\left|\hat{\psi}\left(S^{\mathrm{T}} A^{\mathrm{T}} \omega\right)\right|^{2} d \omega d s d a \\
& =\int_{\Theta_{L}} \int_{\mathbb{R}_{+}} \int_{\mathbb{R}^{d-1}}|\hat{f}(\omega)|^{2} \frac{\operatorname{det}(A)}{a^{d+1}}\left|\hat{\psi}\left(S^{\mathrm{T}} A^{\mathrm{T}} \omega\right)\right|^{2} d s d a d \omega .
\end{aligned}
$$

To continue we need the concrete matrices $A$ and $S$ for the connected shearlet and Toeplitz shearlet groups. We restrict our attention to $\mathbb{S}_{T}^{+}$. The conclusions for $\mathbb{S}^{+}$can be drawn in the same way with slightly simpler substitutions. Then

$$
T_{s}^{\mathrm{T}} A_{a}^{\mathrm{T}} \omega=\left(\begin{array}{ccccc}
1 & 0 & & \cdots & 0 \\
s_{1} & 1 & 0 & & \vdots \\
s_{2} & s_{1} & 1 & 0 & \\
\vdots & \ddots & \ddots & \ddots & 0 \\
s_{d-1} & s_{d-2} & \ldots & s_{1} & 1
\end{array}\right)\left(\begin{array}{r}
a \omega_{1} \\
a \omega_{2} \\
\vdots \\
\vdots \\
a \omega_{d}
\end{array}\right)=\left(\begin{array}{r}
a \omega_{1} \\
s_{1} a \omega_{1}+a \omega_{2} \\
\vdots \\
\vdots \\
s_{d-1} a \omega_{1}+a s_{d-2} \omega_{2}+\ldots+a \omega_{d}
\end{array}\right)
$$


and substituting $\xi_{d}:=s_{d-1} a \omega_{1}+\ldots+a \omega_{d}, \xi_{d-1}:=s_{d-2} a \omega_{1}+\ldots+a \omega_{d-2}, \ldots, \xi_{2}:=s_{1} a \omega_{1}+a \omega_{2}$ we obtain with $d \widetilde{\xi}:=d \xi_{2} \ldots d \xi_{d}$ and $d \widetilde{\xi}=\left(a\left|\omega_{1}\right|\right)^{d-1} d s$ the equality

$$
\begin{aligned}
\int_{\mathbb{S}_{(T)}^{+}}\left|\left\langle f, \psi_{a, s, t}\right\rangle\right|^{2} \frac{d a}{a^{d+1}} d s d t & =\int_{\Theta_{L}} \int_{\mathbb{R}_{+}} \int_{\mathbb{R}^{d-1}}|\hat{f}(\omega)|^{2} a^{-d}\left|\omega_{1}\right|^{-(d-1)}\left|\hat{\psi}\left(a \omega_{1}, \xi_{2}, \ldots, \xi_{d}\right)\right|^{2} d \widetilde{\xi} d a d \omega \\
& =\int_{\Theta_{L}} \int_{\mathbb{R}_{-}} \int_{\mathbb{R}^{d-1}}|\hat{f}(\omega)|^{2}\left|\xi_{1}\right|^{-d}\left|\hat{\psi}\left(\xi_{1}, \xi_{2}, \ldots, \xi_{d}\right)\right|^{2} d \widetilde{\xi} d \xi_{1} d \omega \\
& =\int_{\Theta_{L}}|\hat{f}(\omega)|^{2} d \omega \int_{\Theta_{L}} \frac{|\hat{\psi}(\xi)|^{2}}{\left|\xi_{1}\right|^{d}} d \xi=\|f\|_{L_{2}\left(\Theta_{L}\right)}^{2} \int_{\Theta_{L}} \frac{|\hat{\psi}(\xi)|^{2}}{\left|\xi_{1}\right|^{d}} d \xi
\end{aligned}
$$

where again the left hand side is finite if and only if the right hand side is such. This yields (37) and since there exist functions $\psi \in L_{2}\left(\Theta_{L}\right)$ for which (36) is finite we have verified (30). Next, we show how (37) implies the irreducibility of $\pi$. Suppose by contradiction that it is not irreducible. Then there exist two non-zero functions $f, \psi \in L_{2}\left(\mathbb{R}^{d}\right)$ for which $\left\langle f, \psi_{a, s, t}\right\rangle=0$ as a function of $(a, s, t)$. But then the previous string of equalities yields that the right hand side vanishes. But this in turn implies that either $f=0$ or $\hat{\psi}=0$, a contradiction.

The whole coorbit space setting worked out in [10, 11, 8] for the full shearlet and Toeplitz shearlet groups can now be modified in a straightforward way to the connected groups.

Equivalent representations of $\operatorname{TDS}(d, \mathbb{R})$ and $\operatorname{TDS}_{T}(d, \mathbb{R})$. We want to exploit the isomorphisms $\kappa_{(T)}^{+}$between $\mathbb{S}_{(T)}^{+}$and the subgroups $\operatorname{TDS}_{(T)}(d, \mathbb{R})$ of the symplectic group to define coorbit spaces for the latter subgroups with respect to their metaplectic representations. For this purpose, we define the diffeomorphism

$$
Q: \Theta_{L} \rightarrow \Theta_{L} \quad \text { with } \quad \xi \mapsto Q(\xi):=-\frac{1}{2}\left(\xi_{1}^{2}, \xi_{1} \xi_{2}, \ldots, \xi_{1} \xi_{d}\right)^{\mathrm{T}} .
$$

The inverse of $Q$ is given for $\xi_{1}<0$ by $Q^{-1}(\xi)=\sqrt{2}\left(-\sqrt{-\xi_{1}}, \frac{\xi_{2}}{\sqrt{-\xi_{1}}}, \ldots, \frac{\xi_{d}}{\sqrt{-\xi_{1}}}\right)^{\mathrm{T}}$. Further, the absolute value of the determinants of the Jacobian $\mathcal{J}_{Q}$ of $Q$ and its inverse read

$$
\left|\operatorname{det}\left(\mathcal{J}_{Q}(\xi)\right)\right|=2^{1-d}\left|\xi_{1}\right|^{d} \text { and } \quad\left|\operatorname{det}\left(\mathcal{J}_{Q^{-1}}(\xi)\right)\right|=(\sqrt{2})^{d-2}\left|\xi_{1}\right|^{-\frac{d}{2}} .
$$

Based on $Q$ we can define the isomorphism $\Psi: L_{2}\left(\Theta_{L}\right) \rightarrow L_{2}\left(\Theta_{L}\right)$ as $\Psi=\mathcal{F}^{-1} \hat{\Psi} \mathcal{F}$, where

$$
\hat{\Psi} \hat{f}(\xi)=\left|\operatorname{det}\left(\mathcal{J}_{Q^{-1}}(\xi)\right)\right|^{\frac{1}{2}} \hat{f}\left(Q^{-1}(\xi)\right) .
$$

Its inverse is given by

$$
\hat{\Psi}^{-1} F(\xi)=\left|\operatorname{det}\left(\mathcal{J}_{Q}(\xi)\right)\right|^{\frac{1}{2}} F(Q(\xi)) .
$$

The semi-direct product $\Sigma \rtimes H$ in (17) possesses a (mock) metaplectic representation $\pi^{m}$ on $L_{2}\left(\Theta_{L}\right)$ defined for all $g \in \Sigma \rtimes H$ by

$$
\hat{\pi}^{m}(g) \hat{f}(\xi):=(\operatorname{det} M)^{-\frac{1}{2}} e^{-2 \pi i\langle t, Q(\xi)\rangle} \hat{f}\left(M^{-1} \xi\right), \quad f \in L_{2}\left(\Theta_{L}\right) .
$$

For $\operatorname{TDS}(d, \mathbb{R})$ this metaplectic representation becomes

$$
\hat{\pi}^{m}(a, s, t) \hat{f}(\xi)=a^{\frac{1}{2}-\frac{d}{4}+\frac{\gamma}{2}(d-1)} e^{-2 \pi i\langle t, Q(\xi)\rangle} \hat{f}\left(\widetilde{A}_{a, \gamma}^{-1} \widetilde{S}_{s}^{-1} \xi\right)
$$


with the matrices $\widetilde{A}_{a, \gamma}$ and $\widetilde{S}_{s}$ in (8), and for $\operatorname{TDS}_{T}(d, \mathbb{R})$ just

$$
\hat{\pi}^{m}(a, s, t) \hat{f}(\xi)=a^{\frac{d}{4}} e^{-2 \pi i\langle t, Q(\xi)\rangle} \hat{f}\left(\sqrt{a} T_{s}^{\mathrm{T}} \xi\right) .
$$

We now show that these representations are equivalent to the representations (34) of the connected (Toeplitz) shearlet groups.

Lemma 5.3. Let the isomorphism $\kappa^{+}: \mathbb{S}^{+} \rightarrow \operatorname{TDS}(d, \mathbb{R})$ be given by (10). Then the representation $\pi^{m}$ of $\operatorname{TDS}(d, \mathbb{R})$ in (41) is equivalent to the representation $\pi$ of $\mathbb{S}^{+}$defined by (34) in the sense

$$
\hat{\Psi} \hat{\pi}^{m}\left(\kappa^{+}(\cdot)\right) \hat{\Psi}^{-1}=\mathcal{F} \pi \mathcal{F}^{-1}=\hat{\pi} .
$$

Proof. First we verify that

$$
\begin{aligned}
Q\left(\widetilde{A}_{a, \gamma}^{-1} \widetilde{S}_{s}^{-1} \xi\right) & =Q\left(\left(\begin{array}{cc}
a^{\frac{1}{2}} & 0 \\
s a^{-\frac{1}{2}+\gamma} & a^{-\frac{1}{2}+\gamma} I_{d-1}
\end{array}\right)\left(\begin{array}{c}
\xi_{1} \\
\widetilde{\xi}
\end{array}\right)\right) \\
& =Q\left(a^{\frac{1}{2}} \xi_{1}, s \xi_{1} a^{-\frac{1}{2}+\gamma}+a^{-\frac{1}{2}+\gamma} \widetilde{\xi}\right)=-\frac{1}{2}\left(a \xi_{1}^{2}, s a^{\gamma} \xi_{1}^{2}+a^{\gamma} \xi_{1} \widetilde{\xi}\right) \\
& =-\frac{1}{2} A_{a, \gamma}\left(\begin{array}{c}
\xi_{1}^{2} \\
s \xi_{1}^{2}+\xi_{1} \widetilde{\xi}
\end{array}\right) \\
& =A_{a, \gamma} S_{s}^{\mathrm{T}} Q(\xi) .
\end{aligned}
$$

Then we conclude by (40), the definition of $\hat{\pi}_{m}$ and (44) that

$$
\begin{aligned}
& \left(\hat{\Psi} \hat{\pi}^{m}(a, s, t) \hat{\Psi}^{-1} \hat{\psi}\right)(\xi) \\
& =\left|\operatorname{det}\left(\mathcal{J}_{Q^{-1}}(\xi)\right)\right|^{\frac{1}{2}} \hat{\pi}^{m}(a, s, t)\left(\hat{\Psi}^{-1} \hat{\psi}\right)\left(Q^{-1}(\xi)\right) \\
& =\left|\operatorname{det}\left(\mathcal{J}_{Q^{-1}}(\xi)\right)\right|^{\frac{1}{2}} a^{\frac{1}{2}-\frac{d}{4}+\frac{\gamma}{2}(d-1)}\left(\hat{\Psi}^{-1} \hat{\psi}\right)\left(\widetilde{A}_{a, \gamma}^{-1} \widetilde{S}_{s}^{-1} Q^{-1}(\xi)\right) e^{-2 \pi i\left\langle t, Q\left(Q^{-1}(\xi)\right)\right\rangle} \\
& =\left|\operatorname{det}\left(\mathcal{J}_{Q^{-1}}(\xi)\right)\right|^{\frac{1}{2}} a^{\frac{1}{2}-\frac{d}{4}+\frac{\gamma}{2}(d-1)}\left|\operatorname{det}\left(\mathcal{J}_{Q}\left(\widetilde{A}_{a, \gamma}^{-1} \widetilde{S}_{s}^{-1} Q^{-1}(\xi)\right)\right)\right|^{\frac{1}{2}} \hat{\psi}\left(Q\left(\widetilde{A}_{a, \gamma}^{-1} \widetilde{S}_{s}^{-1} Q^{-1}(\xi)\right)\right) e^{-2 \pi i\langle t, \xi\rangle} \\
& =\left|\operatorname{det}\left(\mathcal{J}_{Q^{-1}}(\xi)\right)\right|^{\frac{1}{2}} a^{\frac{1}{2}-\frac{d}{4}+\frac{\gamma}{2}(d-1)}\left|\operatorname{det}\left(\mathcal{J}_{Q}\left(\widetilde{A}_{a, \gamma}^{-1} \widetilde{S}_{s}^{-1} Q^{-1}(\xi)\right)\right)\right|^{\frac{1}{2}} \hat{\psi}\left(A_{a, \gamma} S_{s}^{\mathrm{T}} \xi\right) e^{-2 \pi i\langle t, \xi\rangle} .
\end{aligned}
$$

By (39) we have

$$
\left|\operatorname{det}\left(\mathcal{J}_{Q^{-1}}(\xi)\right)\right|^{\frac{1}{2}}=(\sqrt{2})^{\frac{d-2}{2}}\left(\sqrt{-\xi_{1}}\right)^{-\frac{d}{2}} .
$$

Simplifying

$$
\begin{aligned}
\left|\operatorname{det}\left(\mathcal{J}_{Q}\left(\widetilde{A}_{a, \gamma}^{-1} \widetilde{S}_{s}^{-1} Q^{-1}(\xi)\right)\right)\right|^{\frac{1}{2}} & =\mid \operatorname{det}\left(\left.\mathcal{J}_{Q}\left(\left(\begin{array}{cc}
a^{\frac{1}{2}} & 0 \\
s a^{-\frac{1}{2}+\gamma} & a^{-\frac{1}{2}+\gamma} I_{d-1}
\end{array}\right)\left(\begin{array}{c}
-\sqrt{-2 \xi_{1}} \\
\frac{\sqrt{2} \xi_{2}}{\sqrt{-\xi_{1}}} \\
\vdots \\
\frac{\sqrt{2} \xi_{d}}{\sqrt{-\xi_{1}}}
\end{array}\right)\right)\right|^{\frac{1}{2}}\right. \\
& =\left(2^{1-d}\left(\sqrt{-2 a \xi_{1}}\right)^{d}\right)^{\frac{1}{2}}=(\sqrt{2})^{(1-d)+\frac{d}{2}} a^{\frac{d}{4}}\left(\sqrt{-\xi_{1}}\right)^{\frac{d}{2}}
\end{aligned}
$$

we obtain further

$$
\begin{aligned}
\left|\operatorname{det}\left(\mathcal{J}_{Q^{-1}}(\xi)\right)\right|^{\frac{1}{2}}\left|\operatorname{det}\left(\mathcal{J}_{Q}\left(\widetilde{A}_{a, \gamma}^{-1} \widetilde{S}_{s}^{-1} Q^{-1}(\xi)\right)\right)\right|^{\frac{1}{2}} & =(\sqrt{2})^{\frac{d-2}{2}}\left(\sqrt{-\xi_{1}}\right)^{-\frac{d}{2}}(\sqrt{2})^{(1-d)+\frac{d}{2}} a^{\frac{d}{4}}\left(\sqrt{-\xi_{1}}\right)^{\frac{d}{2}} \\
& =a^{\frac{d}{4}}
\end{aligned}
$$


and finally

$$
\begin{aligned}
\left(\hat{\Psi} \hat{\pi}^{m}(a, s, t) \hat{\Psi}^{-1} \hat{\psi}\right)(\xi) & =a^{\frac{1}{2}+\frac{\gamma}{2}(d-1)} \hat{\psi}\left(A_{a, \gamma} S_{s}^{\mathrm{T}} \xi\right) e^{-2 \pi i\langle t, \xi\rangle} \\
& =\left|\operatorname{det}\left(A_{a, \gamma}\right)\right|^{\frac{1}{2}} \hat{\psi}\left(A_{a, \gamma} S_{s}^{\mathrm{T}} \xi\right) e^{-2 \pi i\langle t, \xi\rangle} \\
& =\hat{\pi}(a, s, t) \hat{\psi}(\xi) .
\end{aligned}
$$

Equation (43) can be illustrated by the following diagram.

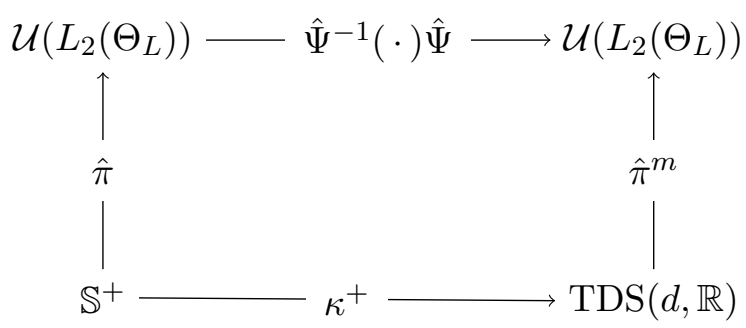

Lemma 5.4. Let the isomorphism $\kappa_{T}^{+}: \mathbb{S}_{T}^{+} \rightarrow \operatorname{TDS}_{T}(d, \mathbb{R})$ be given by (11). Then the representations $\pi^{m}$ of $\operatorname{TDS}_{T}(d, \mathbb{R})$ in (42) and $\pi$ of $\mathbb{S}_{T}^{+}$given by (34) are equivalent in the sense that

$$
\hat{\Psi} \hat{\pi}^{m}\left(\kappa_{T}^{+}(\cdot)\right) \hat{\Psi}^{-1}=\mathcal{F} \pi \mathcal{F}^{-1}=\hat{\pi} .
$$

Proof. By definition of $\sigma(t)$ in (9) we have

$$
\begin{aligned}
\left\langle\sigma\left(t_{1}, \tilde{t}\right) \xi, \xi\right\rangle & =\left\langle\left(\begin{array}{cc}
t_{1} & \frac{1}{2} \tilde{t}^{\mathrm{T}} \\
\frac{1}{2} \tilde{t} & 0
\end{array}\right)\left(\begin{array}{c}
\xi_{1} \\
\widetilde{\xi}
\end{array}\right),\left(\begin{array}{c}
\xi_{1} \\
\widetilde{\xi}
\end{array}\right)\right\rangle=\left\langle\left(\begin{array}{c}
t_{1} \xi_{1}+\frac{1}{2} \tilde{t}^{\mathrm{T}} \widetilde{\xi} \\
\frac{1}{2} \xi_{1} \tilde{t}
\end{array}\right),\left(\begin{array}{c}
\xi_{1} \\
\widetilde{\xi}
\end{array}\right)\right\rangle \\
& =t_{1} \xi_{1}^{2}+\frac{1}{2} \xi_{1} \tilde{t}^{\mathrm{T}} \widetilde{\xi}+\frac{1}{2} \xi_{1} \tilde{t}^{\mathrm{T}} \widetilde{\xi}=t_{1} \xi_{1}^{2}+\xi_{1} \tilde{t}^{\mathrm{T}} \widetilde{\xi} \\
& =-2\langle t, Q(\xi)\rangle .
\end{aligned}
$$

Using (12) we obtain for all $t \in \mathbb{R}^{d}$ that

$$
-2\left\langle t, Q\left(T_{s}^{\mathrm{T}} \xi\right)\right\rangle=\left\langle\sigma\left(t_{1}, \tilde{t}\right) T_{s}^{\mathrm{T}} \xi, T_{s}^{\mathrm{T}} \xi\right\rangle=\left\langle T_{s} \sigma\left(t_{1}, \tilde{t}\right) T_{s}^{\mathrm{T}} \xi, \xi\right\rangle=\left\langle\sigma\left(T_{s} t\right) \xi, \xi\right\rangle
$$

and by the above relation

$$
\left\langle\sigma\left(T_{s} t\right) \xi, \xi\right\rangle=-2\left\langle T_{s} t, Q(\xi)\right\rangle=-2\left\langle t, T_{s}^{\mathrm{T}} Q(\xi)\right\rangle
$$

such that $Q\left(T_{s}^{\mathrm{T}} \xi\right)=T_{s}^{\mathrm{T}} Q(\xi)$ and by definition of $Q$ further

$$
Q\left(\sqrt{a} T_{s}^{\mathrm{T}} \xi\right)=a T_{s}^{\mathrm{T}} Q(\xi) .
$$

Now we can compute

$$
\begin{aligned}
& \left(\hat{\Psi} \hat{\pi}^{m}(a, s, t) \hat{\Psi}^{-1} \hat{\psi}\right)(\xi) \\
& =\left|\operatorname{det}\left(\mathcal{J}_{Q^{-1}}(\xi)\right)\right|^{\frac{1}{2}} \hat{\pi}^{m}(a, s, t)\left(\hat{\Psi}^{-1} \hat{\psi}\right)\left(Q^{-1}(\xi)\right) \\
& =\left|\operatorname{det}\left(\mathcal{J}_{Q^{-1}}(\xi)\right)\right|^{\frac{1}{2}} a^{\frac{d}{4}}\left(\hat{\Psi}^{-1} \hat{\psi}\right)\left(\sqrt{a} T_{s}^{\mathrm{T}} Q^{-1}(\xi)\right) e^{-2 \pi i\left\langle t, Q\left(Q^{-1}(\xi)\right)\right\rangle} \\
& =\left|\operatorname{det}\left(\mathcal{J}_{Q^{-1}}(\xi)\right)\right|^{\frac{1}{2}} a^{\frac{d}{4}}\left|\operatorname{det}\left(\mathcal{J}_{Q}\left(\sqrt{a} T_{s}^{\mathrm{T}} Q^{-1}(\xi)\right)\right)\right|^{\frac{1}{2}} \hat{\psi}\left(Q\left(\sqrt{a} T_{s}^{\mathrm{T}} Q^{-1}(\xi)\right)\right) e^{-2 \pi i\langle t, \xi\rangle} \\
& =\left|\operatorname{det}\left(\mathcal{J}_{Q^{-1}}(\xi)\right)\right|^{\frac{1}{2}} a^{\frac{d}{4}}\left|\operatorname{det}\left(\mathcal{J}_{Q}\left(\sqrt{a} T_{s}^{\mathrm{T}} Q^{-1}(\xi)\right)\right)\right|^{\frac{1}{2}} \hat{\psi}\left(a T_{s}^{\mathrm{T}} \xi\right) e^{-2 \pi i\langle t, \xi\rangle} .
\end{aligned}
$$


Applying

$$
\begin{aligned}
& \left|\operatorname{det}\left(\mathcal{J}_{Q}\left(\sqrt{a} T_{s}^{\mathrm{T}} Q^{-1}(\xi)\right)\right)\right|^{\frac{1}{2}}=\mid \operatorname{det}\left(\left.\mathcal{J}_{Q}\left(\sqrt{a}\left(\begin{array}{cccc}
1 & & & \\
s_{1} & 1 & & \\
\vdots & & \ddots & \\
s_{d-1} & & & 1
\end{array}\right)\left(\begin{array}{c}
-\sqrt{-2 \xi_{1}} \\
\frac{\sqrt{2} \xi_{2}}{\sqrt{-\xi_{1}}} \\
\vdots \\
\frac{\sqrt{2} \xi_{d}}{\sqrt{-\xi_{1}}}
\end{array}\right)\right)\right|^{\frac{1}{2}}\right. \\
& =\left|\operatorname{det}\left(\mathcal{J}_{Q}\left(-\sqrt{-2 a \xi_{1}}, \ldots\right)\right)\right|^{\frac{1}{2}}=\left(2^{1-d}\left(\sqrt{-2 a \xi_{1}}\right)^{d}\right)^{\frac{1}{2}} \\
& =(\sqrt{2})^{(1-d)+\frac{d}{2}} a^{\frac{d}{4}}\left(\sqrt{-\xi_{1}}\right)^{\frac{d}{2}}
\end{aligned}
$$

and (45) we get $\left|\operatorname{det}\left(\mathcal{J}_{Q^{-1}}(\xi)\right)\right|^{\frac{1}{2}}\left|\operatorname{det}\left(\mathcal{J}_{Q}\left(\sqrt{a} S_{s}^{\mathrm{T}} Q^{-1}(\xi)\right)\right)\right|^{\frac{1}{2}}=a^{\frac{d}{4}}$ and finally

$$
\left(\hat{\Psi} \hat{\pi}^{m}(a, s, t) \hat{\Psi}^{-1} \hat{\psi}\right)(\xi)=a^{\frac{d}{2}} \hat{\psi}\left(a T_{s}^{\mathrm{T}} \xi\right) e^{-2 \pi i\langle t, \xi\rangle}=\hat{\pi}(a, s, t) \hat{\psi}(\xi) .
$$

Observe that, in light of (46), a vector $\hat{\psi} \in L_{2}\left(\Theta_{L}\right)$, is admissible for $\hat{\pi}^{m}$ if and only if

$$
\int_{\Theta_{L}} \frac{|\hat{\Psi} \hat{\psi}(\xi)|^{2}}{\left|\xi_{1}\right|^{d}} d \xi<+\infty .
$$

Metaplectic coorbit spaces. Based on the equivalence of the metaplectic representations of the subgroups $\operatorname{TDS}_{(T)}(d, \mathbb{R})$ to square integrable representations of $\mathbb{S}_{(T)}^{+}$we can apply the results from Subsection 5.2 to define coorbit spaces with respect to $\operatorname{TDS}_{(T)}(d, \mathbb{R})$.

Let $\psi \in L_{2}\left(\Theta_{L}\right)$ be an admissible shearlet. Then the transform $\mathcal{S H}_{\psi}: L_{2}\left(\Theta_{L}\right) \rightarrow L_{2}\left(\mathbb{S}_{(T)}^{+}\right)$ defined by

$$
\mathcal{S H}_{\psi}(f)(a, s, t)=\langle f, \pi(a, s, t) \psi\rangle_{L_{2}\left(\Theta_{L}\right)}
$$

is called the continuous (Toeplitz) shearlet transform, whereas the transform $\mathcal{S H}_{\psi}^{m}: L_{2}\left(\Theta_{L}\right) \rightarrow$ $L_{2}\left(\operatorname{TDS}_{(T)}(d)\right)$ defined by

$$
\mathcal{S} \mathcal{H}_{\psi}^{m} f\left(\kappa_{(T)}^{+}(a, s, t)\right)=\left\langle f, \pi^{m}\left(\kappa_{(T)}^{+}(a, s, t)\right) \psi\right\rangle_{L_{2}\left(\Theta_{L}\right)}
$$

is called the metaplectic continuous shearlet transform.

Using the above equivalences the (Toeplitz) shearlet coorbit spaces

$$
\mathcal{S C}_{p, m}:=\left\{f \in \mathcal{H}_{1, w}^{\sim}: \mathcal{S H}_{\psi}(f) \in L_{p, m}\left(\mathbb{S}_{(T)}^{+}\right)\right\}
$$

and the metaplectic shearlet coorbit spaces

$$
\mathcal{S C}_{p, m}^{m}:=\left\{f \in\left(\widetilde{\mathcal{H}}_{1, \widetilde{w}}\right)^{\sim}: \mathcal{S H}_{\psi}^{m}(f) \in L_{p, \widetilde{m}}\left(\operatorname{TDS}_{(T)}(d)\right)\right\}
$$

are diffeomorphic. 


\section{Acknowledgements}

This work has been supported by Deutsche Forschungsgemeinschaft (DFG), Grants DA 360/191 and STE 571/11-1. Some parts of the paper have been written during a stay at the ErwinSchrödinger Institute (ESI), Vienna, Workshop on "Time-Frequency Analysis", January 13-17 2014. Therefore the support of ESI is also acknowledged.

F. De Mari and E. De Vito were partially supported by Progetto PRIN 2010-2011 "Varietà reali e complesse: geometria, topologia e analisi armonica". They are members of the Gruppo Nazionale per l'Analisi Matematica, la Probabilità e le loro Applicazioni (GNAMPA) of the Istituto Nazionale di Alta Matematica (INdAM).

S. Dahlke, S. Häuser, G. Steidl and G. Teschke were partially supported by DAAD Project 57056121, "Hochschuldialog mit Südeuropa 2013".

\section{Appendix}

In the following we list the Lie brackets of the canonical matrices $D \in \mathcal{N}$ with the basis matrices $X_{\nu}, \nu \in \triangle$, and $H_{1,0}, H_{0,1}$ from the root space decomposition of $\mathfrak{s p}(2, \mathbb{R})$ and the matrices $M_{\Gamma}$ defined by (29).

Case 1. For $D_{1}=a_{1} H_{1,0}+a_{2} H_{0,1}$ we obtain

$$
\begin{array}{llll}
{\left[D_{1}, X_{\alpha}\right]} & =\left(a_{1}-a_{2}\right) X_{\alpha}, & {\left[D_{1}, X_{-\alpha}\right]} & =-\left(a_{1}-a_{2}\right) X_{-\alpha}, \\
{\left[D_{1}, X_{\beta}\right]} & =2 a_{2} X_{\beta}, & {\left[D_{1}, X_{-\beta}\right]} & =-2 a_{2} X_{-\beta}, \\
{\left[D_{1}, X_{\alpha+\beta}\right]} & =\left(a_{1}+a_{2}\right) X_{\alpha+\beta}, & {\left[D_{1}, X_{-\alpha-\beta}\right]} & =-\left(a_{1}+a_{2}\right) X_{-\alpha-\beta}, \\
{\left[D_{1}, X_{2 \alpha+\beta}\right]} & =2 a_{1} X_{2 \alpha+\beta}, & {\left[D_{1}, X_{-2 \alpha-\beta}\right]} & =-2 a_{1} X_{-2 \alpha-\beta}, \\
{\left[D_{1}, H_{1,0}\right]} & =0, & {\left[D_{1}, H_{0,1}\right]} & =0
\end{array}
$$

and $M_{\Gamma}$ is a diagonal matrix with entries

$$
\left(a_{1}-a_{2}-\Gamma, 2 a_{2}-\Gamma, a_{1}+a_{2}-\Gamma, 2 a_{1}-\Gamma, a_{2}-a_{1}-\Gamma,-2 a_{2}-\Gamma,-a_{2}-a_{1}-\Gamma,-2 a_{1}-\Gamma,-\Gamma,-\Gamma\right),
$$

where $a_{1} \geq a_{2} \geq 0$.

Case 2. For $D_{2}=X_{-\alpha}+a H_{1,0}+a H_{0,1}$ we obtain

$$
\begin{array}{llll}
{\left[D_{2}, X_{\alpha}\right]} & =H_{1,0}-H_{0,1}, & {\left[D_{2}, X_{-\alpha}\right]} & =0, \\
{\left[D_{2}, X_{\beta}\right]} & =2 a X_{\beta}, & {\left[D_{2}, X_{-\beta}\right]} & =-2 a X_{-\beta}+X_{-\alpha-\beta}, \\
{\left[D_{2}, X_{\alpha+\beta}\right]} & =-2 X_{\beta}+2 a X_{\alpha+\beta}, & {\left[D_{2}, X_{-\alpha-\beta}\right]} & =-2 a X_{-\alpha-\beta}+X_{-2 \alpha-\beta}, \\
{\left[D_{2}, X_{2 \alpha+\beta}\right]} & =-2 X_{\alpha+\beta}+2 a X_{2 \alpha+\beta}, & {\left[D_{2}, X_{-2 \alpha-\beta}\right]} & =-2 a X_{-2 \alpha-\beta}, \\
{\left[D_{2}, H_{1,0}\right]} & =X_{-\alpha}, & {\left[D_{2}, H_{0,1}\right]} & =-X_{-\alpha}
\end{array}
$$


and

$$
M_{\Gamma}=\left(\begin{array}{cccccccccc}
-\Gamma & 0 & 0 & 0 & 0 & 0 & 0 & 0 & 0 & 0 \\
0 & 2 a-\Gamma & -2 & 0 & 0 & 0 & 0 & 0 & 0 & 0 \\
0 & 0 & 2 a-\Gamma & -2 & 0 & 0 & 0 & 0 & 0 & 0 \\
0 & 0 & 0 & 2 a-\Gamma & 0 & 0 & 0 & 0 & 0 & 0 \\
0 & 0 & 0 & 0 & -\Gamma & 0 & 0 & 0 & 1 & -1 \\
0 & 0 & 0 & 0 & 0 & -2 a-\Gamma & 0 & 0 & 0 & 0 \\
0 & 0 & 0 & 0 & 0 & 1 & -2 a-\Gamma & 0 & 0 & 0 \\
0 & 0 & 0 & 0 & 0 & 0 & 1 & -2 a-\Gamma & 0 & 0 \\
1 & 0 & 0 & 0 & 0 & 0 & 0 & 0 & -\Gamma & 0 \\
-1 & 0 & 0 & 0 & 0 & 0 & 0 & 0 & 0 & -\Gamma
\end{array}\right)
$$

with

$$
\operatorname{det} M_{\Gamma}=\Gamma^{4}(\Gamma-2 a)^{3}(\Gamma+2 a)^{3}, \quad a \geq 0 .
$$

Case 3. For $D_{3}=b X_{\alpha}+b X_{-\alpha}+a H_{1,0}+a H_{0,1}$ we obtain

$$
\begin{aligned}
& {\left[D_{3}, X_{\alpha}\right]=b H_{1,0}-b H_{0,1} \text {, }} \\
& {\left[D_{3}, X_{\beta}\right]=2 a X_{\beta}+b X_{\alpha+\beta},} \\
& {\left[D_{3}, X_{-\alpha}\right]=-b H_{1,0}+b H_{0,1},} \\
& {\left[D_{3}, X_{\alpha+\beta}\right]=-2 b X_{\beta}+2 a X_{\alpha+\beta}+b X_{2 \alpha+\beta} \text {, }} \\
& {\left[D_{3}, X_{-\beta}\right]=-2 a X_{-\beta}+b X_{-\alpha-\beta} \text {, }} \\
& {\left[D_{3}, X_{-\alpha-\beta}\right]=-2 b X_{-\beta}-2 a X_{-\alpha-\beta}} \\
& {\left[D_{3}, X_{2 \alpha+\beta}\right]=-2 b X_{\alpha+\beta}+2 a X_{2 \alpha+\beta},} \\
& +b X_{-2 \alpha-\beta} \text {, } \\
& {\left[D_{3}, H_{1,0}\right] \quad=-b X_{\alpha}+b X_{-\alpha} \text {, }} \\
& {\left[D_{3}, X_{-2 \alpha-\beta}\right]=-2 b X_{-\alpha-\beta}-2 a X_{-2 \alpha-\beta} \text {, }} \\
& {\left[D_{3}, H_{0,1}\right]=b X_{\alpha}-b X_{-\alpha}}
\end{aligned}
$$

and

$$
M_{\Gamma}=\left(\begin{array}{cccccccccc}
-\Gamma & 0 & 0 & 0 & 0 & 0 & 0 & 0 & -b & b \\
0 & 2 a-\Gamma & -2 b & 0 & 0 & 0 & 0 & 0 & 0 & 0 \\
0 & b & 2 a-\Gamma & -2 b & 0 & 0 & 0 & 0 & 0 & 0 \\
0 & 0 & b & 2 a-\Gamma & 0 & 0 & 0 & 0 & 0 & 0 \\
0 & 0 & 0 & 0 & -\Gamma & 0 & 0 & 0 & b & -b \\
0 & 0 & 0 & 0 & 0 & -2 a-\Gamma & -2 b & 0 & 0 & 0 \\
0 & 0 & 0 & 0 & 0 & b & -2 a-\Gamma & -2 b & 0 & 0 \\
0 & 0 & 0 & 0 & 0 & 0 & b & -2 a-\Gamma & 0 & 0 \\
b & 0 & 0 & 0 & -b & 0 & 0 & 0 & -\Gamma & 0 \\
-b & 0 & 0 & 0 & b & 0 & 0 & 0 & 0 & -\Gamma
\end{array}\right)
$$

with

$$
\operatorname{det} M_{\Gamma}=\Gamma^{2}\left(\Gamma^{2}+4 b^{2}\right)(\Gamma-2 a)(\Gamma+2 a)\left((\Gamma-2 a)^{2}+4 b^{2}\right)\left((\Gamma++2 a)^{2}+4 b^{2}\right), \quad a, b>0 .
$$

Case 4. For $D_{4}=\varepsilon X_{\alpha+\beta}-X_{-\alpha}-\frac{\varepsilon}{2} X_{-2 \alpha-\beta}$ we obtain

$$
\begin{array}{llll}
{\left[D_{4}, X_{\alpha}\right]} & =-\varepsilon X_{2 \alpha+\beta}-\varepsilon X_{-\alpha-\beta}-H_{1,0}+H_{0,1}, & {\left[D_{4}, X_{-\alpha}\right]} & =2 \varepsilon X_{\beta}, \\
{\left[D_{4}, X_{\beta}\right]=0,} & {\left[D_{4}, X_{-\beta}\right]} & =-\varepsilon X_{\alpha}-X_{-\alpha-\beta}, \\
{\left[D_{4}, X_{\alpha+\beta}\right]=2 X_{\beta}+\varepsilon X_{-\alpha},} & {\left[D_{4}, X_{-\alpha-\beta}\right]=} & -X_{-2 \alpha-\beta}-\varepsilon H_{1,0} \\
& & -\varepsilon H_{0,1}, \\
{\left[D_{4}, X_{2 \alpha+\beta}\right]=2 X_{\alpha+\beta}-2 \varepsilon H_{1,0},} & {\left[D_{4}, X_{-2 \alpha-\beta}\right]=} & 2 \varepsilon X_{-\alpha}, \\
{\left[D_{4}, H_{1,0}\right]=-\varepsilon X_{\alpha+\beta}-X_{-\alpha}-\varepsilon X_{-2 \alpha-\beta},} & {\left[D_{4}, H_{0,1}\right]} & =-\varepsilon X_{\alpha+\beta}+X_{-\alpha}
\end{array}
$$


and

$$
M_{\Gamma}=\left(\begin{array}{cccccccccc}
-\Gamma & 0 & 0 & 0 & 0 & -\varepsilon & 0 & 0 & 0 & 0 \\
0 & -\Gamma & 2 & 0 & 2 \varepsilon & 0 & 0 & 0 & 0 & 0 \\
0 & 0 & -\Gamma & 2 & 0 & 0 & 0 & 0 & -\varepsilon & -\varepsilon \\
-\varepsilon & 0 & 0 & -\Gamma & 0 & 0 & 0 & 0 & 0 & 0 \\
0 & 0 & \varepsilon & 0 & -\Gamma & 0 & 0 & 2 \varepsilon & -1 & 1 \\
0 & 0 & 0 & 0 & 0 & -\Gamma & 0 & 0 & 0 & 0 \\
-\varepsilon & 0 & 0 & 0 & 0 & -1 & -\Gamma & 0 & 0 & 0 \\
0 & 0 & 0 & 0 & 0 & 0 & -1 & -\Gamma & -\varepsilon & 0 \\
-1 & 0 & 0 & -2 \varepsilon & 0 & 0 & -\varepsilon & 0 & -\Gamma & 0 \\
1 & 0 & 0 & 0 & 0 & 0 & -\varepsilon & 0 & 0 & -\Gamma
\end{array}\right)
$$

with $\operatorname{det} M_{\Gamma}=\Gamma^{10}$.

Case 5. For $D_{5}=\frac{\varepsilon}{2} X_{2 \alpha+\beta}+\frac{b^{2} \varepsilon}{2} X_{-2 \alpha-\beta}+a H_{0,1}$ we obtain

$$
\begin{array}{llll}
{\left[D_{5}, X_{\alpha}\right]} & =-a X_{\alpha}+b^{2} \varepsilon X_{-\alpha-\beta}, & {\left[D_{5}, X_{-\alpha}\right]} & =\varepsilon X_{\alpha+\beta}, \\
{\left[D_{5}, X_{\beta}\right]} & =2 a X_{\beta}, & {\left[D_{5}, X_{-\beta}\right]} & =-2 a X_{-\beta}, \\
{\left[D_{5}, X_{\alpha+\beta}\right]} & =a X_{\alpha+\beta}-b^{2} \varepsilon X_{-\alpha}, & {\left[D_{5}, X_{-\alpha-\beta}\right]} & =-\varepsilon X_{\alpha}-a X_{-\alpha-\beta}, \\
{\left[D_{5}, X_{2 \alpha+\beta}\right]} & =2 b^{2} \varepsilon H_{1,0}, & {\left[D_{5}, X_{-2 \alpha-\beta}\right]} & =-2 \varepsilon H_{1,0}, \\
{\left[D_{5}, H_{1,0}\right]} & =-\varepsilon X_{2 \alpha+\beta}+b^{2} \varepsilon X_{-2 \alpha-\beta}, & {\left[D_{5}, H_{0,1}\right],} & =0
\end{array}
$$

and

$$
M_{\Gamma}=\left(\begin{array}{cccccccccc}
-a-\Gamma & 0 & 0 & 0 & 0 & 0 & -\varepsilon & 0 & 0 & 0 \\
0 & 2 a-\Gamma & 0 & 0 & 0 & 0 & 0 & 0 & 0 & 0 \\
0 & 0 & a-\Gamma & 0 & \varepsilon & 0 & 0 & 0 & 0 & 0 \\
0 & 0 & 0 & -\Gamma & 0 & 0 & 0 & 0 & -\varepsilon & 0 \\
0 & 0 & -b^{2} \varepsilon & 0 & a-\Gamma & 0 & 0 & 0 & 0 & 0 \\
0 & 0 & 0 & 0 & 0 & -2 a-\Gamma & 0 & 0 & 0 & 0 \\
b^{2} \varepsilon & 0 & 0 & 0 & 0 & 0 & -a-\Gamma & 0 & 0 & 0 \\
0 & 0 & 0 & 0 & 0 & 0 & 0 & -\Gamma & b^{2} \varepsilon & 0 \\
0 & 0 & 0 & 2 b^{2} \varepsilon & 0 & 0 & 0 & -2 \varepsilon & -\Gamma & 0 \\
0 & 0 & 0 & 0 & 0 & 0 & 0 & 0 & 0 & -\Gamma
\end{array}\right)
$$

with

$\operatorname{det} M_{\Gamma}=\Gamma^{2}(\Gamma-2 a)(\Gamma+2 a)\left(\Gamma^{2}+4 b^{2}\right)\left((\Gamma-a)^{2}+b^{2}\right)\left(\left(\Gamma^{2}+a\right)^{2}+b^{2}\right), \quad a, b \geq 0$.

Case 6. For $D_{6}=\eta X_{\beta}+\frac{\varepsilon}{2} X_{2 \alpha+\beta}+b_{2}^{2} \eta X_{-\beta}+\frac{b_{1}^{2} \varepsilon}{2} X_{-2 \alpha-\beta}$ we obtain

$$
\begin{aligned}
& {\left[D_{6}, X_{\alpha}\right]=-\eta X_{\alpha+\beta}+b_{1}^{2} \varepsilon X_{-\alpha-\beta}, \quad\left[D_{6}, X_{-\alpha}\right]=\varepsilon X_{\alpha+\beta}-b_{2}^{2} \eta X_{-\alpha-\beta},} \\
& {\left[D_{6}, X_{\beta}\right] \quad=b_{2}^{2} \eta H_{0,1}, \quad\left[D_{6}, X_{-\beta}\right]=-\eta H_{0,1},} \\
& {\left[D_{6}, X_{\alpha+\beta}\right]=b_{2}^{2} \eta X_{\alpha}-b_{1}^{2} \varepsilon X_{-\alpha}, \quad\left[D_{6}, X_{-\alpha-\beta}\right]=-\varepsilon X_{\alpha}+\eta X_{-\alpha} \text {, }} \\
& {\left[D_{6}, X_{2 \alpha+\beta}\right]=2 b_{1}^{2} \varepsilon H_{1,0}, \quad\left[D_{6}, X_{-2 \alpha-\beta}\right]=-2 \varepsilon H_{1,0},} \\
& {\left[D_{6}, H_{1,0}\right]=-\varepsilon X_{2 \alpha+\beta}+b_{1}^{2} \varepsilon X_{-2 \alpha-\beta}, \quad\left[D_{6}, H_{0,1}\right]=-2 \eta X_{\beta}+2 b_{2}^{2} \eta X_{-\beta}}
\end{aligned}
$$


and

$$
M_{\Gamma}=\left(\begin{array}{cccccccccc}
-\Gamma & 0 & b_{2}^{2} \eta & 0 & 0 & 0 & -\varepsilon & 0 & 0 & 0 \\
0 & -\Gamma & 0 & 0 & 0 & 0 & 0 & 0 & 0 & -2 \eta \\
-\eta & 0 & -\Gamma & 0 & \varepsilon & 0 & 0 & 0 & 0 & 0 \\
0 & 0 & 0 & -\Gamma & 0 & 0 & 0 & 0 & -\varepsilon & 0 \\
0 & 0 & -b_{1}^{2} \varepsilon & 0 & -\Gamma & 0 & \eta & 0 & 0 & 0 \\
0 & 0 & 0 & 0 & 0 & -\Gamma & 0 & 0 & 0 & 2 b_{2}^{2} \eta \\
b_{1}^{2} \varepsilon & 0 & 0 & 0 & -b_{2}^{2} \eta & 0 & -\Gamma & 0 & 0 & 0 \\
0 & 0 & 0 & 0 & 0 & 0 & 0 & -\Gamma & b_{1}^{2} \varepsilon & 0 \\
0 & 0 & 0 & 2 b_{1}^{2} \varepsilon & 0 & 0 & 0 & -2 \varepsilon & -\Gamma & 0 \\
0 & b_{2}^{2} \eta & 0 & 0 & 0 & -\eta & 0 & 0 & 0 & -\Gamma
\end{array}\right)
$$

with

$$
\operatorname{det} M_{\Gamma}=\Gamma^{2}\left(\Gamma^{2}+4 b_{2}^{2}\right)\left(\Gamma^{2}+4 b_{1}^{2}\right)\left(\Gamma^{2}+\left(b_{1}-b_{2}\right)^{2}\right)\left(\Gamma^{2}+\left(b_{1}+b_{2}\right)^{2}\right), \quad b_{1} \geq b_{2} \geq 0 .
$$

Case 7. For $D_{7}=-X_{\alpha}-\varepsilon X_{\beta}+\frac{\varepsilon}{2 b^{2}} X_{2 \alpha+\beta}-b^{2} X_{-\alpha}$ and

$$
\begin{aligned}
& {\left[D_{7}, X_{\alpha}\right]=-\varepsilon X_{\alpha+\beta}-b^{2} H_{1,0}+b^{2} H_{0,1}, \quad\left[D_{7}, X_{-\alpha}\right] \quad=\frac{\varepsilon}{b^{2}} X_{\alpha+\beta}+H_{1,0}-H_{0,1},} \\
& {\left[D_{7}, X_{\beta}\right]=-X_{\alpha+\beta}, \quad\left[D_{7}, X_{-\beta}\right]=-b^{2} X_{-\alpha-\beta}-\varepsilon H_{0,1} \text {, }} \\
& {\left[D_{7}, X_{\alpha+\beta}\right]=2 b^{2} X_{\beta}-X_{2 \alpha+\beta}, \quad\left[D_{7}, X_{-\alpha-\beta}\right]=-\frac{\varepsilon}{b^{2}} X_{\alpha}+\varepsilon X_{-\alpha}} \\
& {\left[D_{7}, X_{2 \alpha+\beta}\right]=2 b^{2} X_{\alpha+\beta}, \quad\left[D_{7}, X_{-2 \alpha-\beta}\right]=\begin{array}{l}
+X_{-\alpha-\beta}-\frac{2 \varepsilon}{b^{2}} H_{1,0}, \\
{\left[D_{7}, X_{1,0}, X_{-\alpha} X_{-\alpha}\right.}
\end{array}} \\
& {\left[D_{7}, H_{1,0}\right]=X_{\alpha}-\frac{\varepsilon}{b^{2}} X_{2 \alpha+\beta}-b^{2} X_{-\alpha}, \quad\left[D_{7}, H_{0,1}\right]=-X_{\alpha}-2 \varepsilon X_{\beta}+b^{2} X_{-\alpha}}
\end{aligned}
$$

and

$$
M_{\Gamma}=\left(\begin{array}{cccccccccc}
-\Gamma & 0 & 0 & 0 & 0 & 0 & -\frac{\varepsilon}{b^{2}} & 0 & 1 & -1 \\
0 & -\Gamma & 2 b^{2} & 0 & 0 & 0 & 0 & 0 & 0 & -2 \varepsilon \\
-\varepsilon & -1 & -\Gamma & 2 b^{2} & \frac{\varepsilon}{b^{2}} & 0 & 0 & 0 & 0 & 0 \\
0 & 0 & -1 & -\Gamma & 0 & 0 & 0 & 0 & -\frac{\varepsilon}{b^{2}} & 0 \\
0 & 0 & 0 & 0 & -\Gamma & 0 & \varepsilon & 0 & -b^{2} & b^{2} \\
0 & 0 & 0 & 0 & 0 & -\Gamma & 2 & 0 & 0 & 0 \\
0 & 0 & 0 & 0 & 0 & -b^{2} & -\Gamma & 2 & 0 & 0 \\
0 & 0 & 0 & 0 & 0 & 0 & -b^{2} & -\Gamma & 0 & 0 \\
-b^{2} & 0 & 0 & 0 & 1 & 0 & 0 & -\frac{2 \varepsilon}{b^{2}} & -\Gamma & 0 \\
b^{2} & 0 & 0 & 0 & -1 & -\varepsilon & 0 & 0 & 0 & -\Gamma
\end{array}\right)
$$

with $\operatorname{det} M_{\Gamma}=\Gamma^{4}\left(\Gamma^{2}+4 b^{2}\right)^{3}, b>0$.

\section{References}

[1] V. I. Arnol'd. Mathematical Methods of Classical Mechanics, volume 60 of Graduate Texts in Mathematics. Springer, 1989.

[2] E. Cordero, F. De Mari, K. Nowak, and A. Tabacco. Analytic features of reproducing groups for the metaplectic representation. Journal of Fourier Analysis and Applications, 12(2):157-180, 2006.

[3] E. Cordero, F. De Mari, K. Nowak, and A. Tabacco. Reproducing groups for the metaplectic representation. In P. Boggiatto, L. Rodino, J. Toft, and M. W. Wong, editors, Pseudo-Differential Operators and Related Topics, volume 12 of Operator Theory: Advances and Applications, pages 227-244. Birkhäuser Basel, 2006. 
[4] E. Cordero, F. De Mari, K. Nowak, and A. Tabacco. Dimensional upper bounds for admissible subgroups for the metaplectic representation. Mathematische Nachrichten, 283(7):982993, 2010.

[5] W. Czaja and E. J. King. Isotropic shearlet analogs for $L^{2}\left(\mathbb{R}^{k}\right)$ and localization operators. Numerical Functional Analysis and Optimization, 33(7-9):872-905, 2012.

[6] W. Czaja and E. J. King. Anisotropic shearlet transforms for $L^{2}\left(\mathbb{R}^{k}\right)$. Mathematische Nachrichten, 287(8-9):903-916, 2014.

[7] S. Dahlke, S. Häuser, G. Steidl, and G. Teschke. Shearlet coorbit spaces: traces and embeddings in higher dimensions. Monatshefte für Mathematik, 169(1):15-32, 2013.

[8] S. Dahlke, S. Häuser, and G. Teschke. Coorbit space theory for the Toeplitz shearlet transform. International Journal of Wavelets, Multiresolution and Information Processing, $10(4), 2012$.

[9] S. Dahlke, G. Kutyniok, P. Maass, C. Sagiv, H.-G. Stark, and G. Teschke. The uncertainty principle associated with the continuous shearlet transform. International Journal on Wavelets Multiresolution and Information Processing, 6:157-181, 2008.

[10] S. Dahlke, G. Kutyniok, G. Steidl, and G. Teschke. Shearlet coorbit spaces and associated Banach frames. Applied and Computational Harmonic Analysis, 27(2):195-214, 2009.

[11] S. Dahlke, G. Steidl, and G. Teschke. The continuous shearlet transform in arbitrary space dimensions. Journal of Fourier Analysis and Applications, 16(3):340-364, 2010.

[12] S. Dahlke and G. Teschke. The continuous shearlet transform in higher dimensions: variations of a theme. In C. W. Danellis, editor, Group Theory: Classes, Representations and Connections, and Applications, pages 167-175. Nova Science Pub Inc, 2010.

[13] F. De Mari and E. De Vito. Admissible vectors for mock metaplectic representations. Applied Computational Harmonic Analysis, 34(2):163-200, 2013.

[14] H. G. Feichtinger and K. Gröchenig. A unified approach to atomic decompositions via integrable group representations. In Function spaces and applications, (Lund, 1986), Vol. 1302 of Lecture Notes in Mathematics, pages 52-73. Springer, 1988.

[15] H. G. Feichtinger and K. Gröchenig. Banach spaces related to integrable group representations and their atomic decompositions, Part I. Journal of Functional Analysis, 86(2):307-340, 1989.

[16] H. G. Feichtinger and K. Gröchenig. Banach spaces related to integrable group representations and their atomic decompositions. Part II. Monatshefte für Mathematik, 108(2):129$148,1989$.

[17] G. B. Folland. A Course in Abstract Harmonic Analysis. CRC Press, 1995.

[18] K. Gröchenig. Describing functions: atomic decompositions versus frames. Monatshefte für Mathematik, 112(1):1-42, 1991.

[19] K. Gröchenig. Foundations of Time-Frequency Analysis. Birkhäuser, 2001.

[20] K. Gröchenig and M. Piotrowski. Molecules in coorbit spaces and boundedness of operators. Studia Mathematica, 192(1):61-77, 2009. 
[21] P. Grohs. Continuous shearlet frames and resolution of the wavefront set. Monatshefte für Mathematik, 164(4):393-426, 2011.

[22] K. Guo and D. Labate. Characterization and analysis of edges using the continuous shearlet transform. SIAM Journal on Imaging Sciences, 2(3):959-986, 2009.

[23] K. Guo, D. Labate, and W. Lim. Edge analysis and identification using the continuous shearlet transform. Applied and Computational Harmonic Analysis, 27(1):24-46, 2009.

[24] S. Häuser. Shearlet Coorbit Spaces, Shearlet Transforms and Applications in Imaging. Dissertation, TU Kaiserslautern, 2014.

[25] S. Häuser and G. Steidl. Convex multiclass segmentation with shearlet regularization. International Journal of Computer Mathematics, 90(1):62-81, 2013.

[26] E. Kaniuth and K. F. Taylor. Induced Representations of Locally Compact Groups, volume 197 of Cambridge Tracts in Mathematics. Cambridge University Press, 2013.

[27] E. J. King. Wavelet and frame theory: frame bound gaps, generalized shearlets, Grassmannian fusion frames, and p-adic wavelets. Dissertation, University of Maryland, College Park, 2009.

[28] E. J. King, G. Kutyniok, and W.-Q. Lim. Image inpainting: theoretical analysis and comparison of algorithms. In D. Van De Ville, V. K. Goyal, and M. Papadakis, editors, SPIE Optical Engineering + Applications, page 885802. International Society for Optics and Photonics, Sept. 2013.

[29] A. W. Knapp. Lie Groups beyond an Introduction, volume 140. Birkhäuser, 2002.

[30] G. Kutyniok and D. Labate, editors. Shearlets: Multiscale Analysis for Multivariate Data. Springer, 2012.

[31] R. S. S. Laugesen, N. Weaver, G. L. Weiss, and E. N. Wilson. A characterization of the higher dimensional groups associated with continuous wavelets. Journal of Geometric Analysis, 12(1):89-102, 2002.

[32] E. Schulz and K. F. Taylor. Extensions of the Heisenberg group and wavelet analysis in the plane. In Spline Functions and the Theory of Wavelets (Montreal, PQ, 1996), volume 18 of CRM Proceedings and Lecture Notes, pages 217-225, American Mathematical Society.

[33] J. Williamson. On the algebraic problem concerning the normal forms of linear dynamical systems. American Journal of Mathematics, 58(1):141-163, 1936. 